\title{
Coercive inequalities in higher-dimensional anisotropic heisenberg group
}

\author{
Esther Bou Dagher ${ }^{1}$ D $\cdot$ Bogusław Zegarliński $^{1}$
}

Received: 8 May 2021 / Revised: 27 September 2021 / Accepted: 3 October 2021 /

Published online: 1 November 2021

(c) The Author(s) 2021

\section{Abstract}

In the setting of higher-dimensional anisotropic Heisenberg group, we compute the fundamental solution for the sub-Laplacian, and we prove Poincaré and $\beta$-Logarithmic Sobolev inequalities for measures as a function of this fundamental solution.

Keywords Poincaré inequality $\cdot$ Logarithmic Sobolev inequality $\cdot$ Anisotropic Heisenberg group $\cdot$ Sub-gradient $\cdot$ Fundamental solution $\cdot$ Probability measures

\section{Contents}

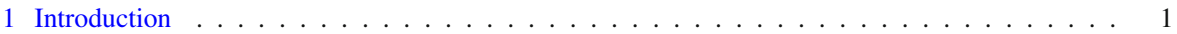

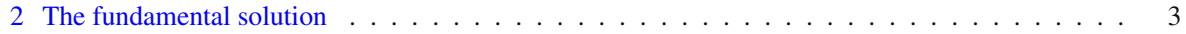

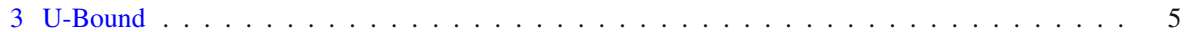

$4 q$-Poincaré and $\beta$-logarithmic Sobolev inequalities . . . . . . . . . . . . . . . . . . . . . . . . . . 13

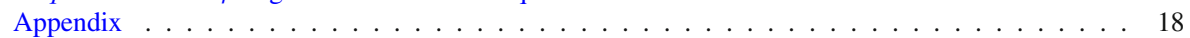

Derivation of the formula for homogeneous norm $N \ldots \ldots \ldots \ldots$

Proof of Lemma 2: Bounds for $|\nabla N|$ and $x \cdot \nabla N \ldots \ldots \ldots \ldots \ldots \ldots$

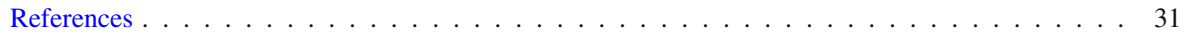

\section{Introduction}

Folland showed in [14] that on a Carnot group $\mathbb{G}$, the sub-Laplacian $\triangle:=\sum_{i=1}^{n} X_{i}^{2}$ admits a unique fundamental solution $N^{2-Q}$, i.e.

$$
\triangle N^{2-Q}=\delta
$$

$\triangle \quad$ Esther Bou Dagher

esther.bou-dagher17@imperial.ac.uk

Bogusław Zegarliński

b.zegarlinski@imperial.ac.uk

1 Department of Mathematics, Imperial College London, 180 Queen's Gate, London SW7 2AZ, UK

Birkhäuser 
where $\delta$ is the delta-distribution at the unit element of $\mathbb{G}, X_{1}, \ldots, X_{n}$ are the Jacobian generators of $\mathbb{G}, Q$ is the homogeneous dimension, and $N$ is a homogeneous norm on $\mathbb{G}$.

Balogh and Tyson [2] introduced the concept of polarizable Carnot groups defined by the condition that $N$ is $\infty$-harmonic in $\mathbb{G} \backslash\{0\}$, i.e. for $\nabla:=\left(X_{i}\right)_{1 \leq i \leq n}$,

$$
\triangle_{\infty} N:=\frac{1}{2}<\nabla\left(|\nabla N|^{2}\right), \nabla N>=0 \quad \text { in } \mathbb{G} \backslash\{0\} .
$$

They have shown that using the $\infty$-harmonicity of $N$ one can provide a procedure to construct polar coordinates of special type where the curves passing through the points on the unit sphere $\{N=1\}$ are horizontal.

Moreover, they showed in [2] that the fundamental solution of the $p$-sub-Laplacian can be expressed as the fundamental solution $N$ of the sub-Laplacian, proved capacity formulas, and produced sharp constants for the Moser-Trudinger inequality (which was established by Saloff-Coste [33] in the setting of Carnot groups but without sharp constants). In settings related to polarizable Carnot groups, many authors showed Hardy-type inequalities as a function of $N$ (see $[12,13,15,16,22,35]$ ), proved Rellichtype inequalities (see $[22,36]$ ), and studied Fuglede's $p$-module of system of measures [9].

For the time being, there is no a classification of polarizable Carnot groups, and the only examples till now are Euclidean spaces and Heisenberg-type groups. In addition, the concept of a polarizable Carnot group is a delicate one in the sense that under a small perturbation of the Lie algebra, the group is no longer polarizable. Balogh and Tyson provided in [2] the anisotropic Heisenberg group in $\mathbb{R}^{4}$ as a counterexample with the following generators of the Lie algebra: $X=\frac{\partial}{\partial x}+2 a y \cdot \frac{\partial}{\partial t}, Y=\frac{\partial}{\partial y}-2 a x \cdot \frac{\partial}{\partial t}$, $Z=\frac{\partial}{\partial z}-2 w \cdot \frac{\partial}{\partial t}$, and $W=\frac{\partial}{\partial w}-2 z \cdot \frac{\partial}{\partial t}$, where $a=\frac{1}{2}$. (Note that if $a=1$, we have the polarizable Heisenberg group.) To show (1.1) does not hold true for the anisotropic Heisenberg group, they computed explicitly the fundamental solution of the sub-Laplacian using Beals et al.'s [3] explicit integral representation for the fundamental solution in the setting of general step-two Carnot groups.

Recently, Bieske [4] revisited this counterexample and proved that under a change of the inner product imposed on the vectors in the Lie Algebra, which now requires the generators to be orthogonal instead of orthonormal, the anisotropic Heisenberg group is turned into a group of Heisenberg-type i.e. it is now polarizable!

The goal of this paper is to study coercive inequalities such as the $q$-Poincaré inequality and the $\beta$-Logarithmic Sobolev inequality in the setting of the anisotropic Heisenberg group with respect to measures as a function of the explicit fundamental solution. We will first show the computations, that were partially omitted in [2] in the $\mathbb{R}^{5}$ setting, and use that to get an explicit fundamental solution for higher dimensions.

In the setting of nilpotent Lie groups, heat kernel estimates have been used to get coercive inequalities [1,11,23-28,30,34]. In our setting, we do not use heat kernel estimates; instead, we study coercive inequalities involving sub-gradients and probability measures depending on the group. An approach to study such problems was pioneered in [18]. This approach relies on the method of $U$-bounds, where $U$ stands for the potential. It was later used by Inglis to get the Poincaré inequality in the setting of 
the Heisenberg-type group with the measure as a function of the Kaplan distance [19]. In addition, Chatzakou et al. use this U-Bound method to get the Poincaré inequality in the setting of the Engel-type group with the measure as a function of some quasihomegenous norm [10]. This approach was also used by the authors of this paper in [8] to get the $q$-Poincaré inequality and the $\phi$-Logarithmic Sobolev inequality in the setting of step-two Carnot groups with measures as a function of a generalised Kaplan norm [8].

The method of [18] to get coercive inequalities relies on the study of so-called U-bounds, i.e. bounds of the following type

$$
\int|f|^{q} U d \mu \leq C \int|\nabla f|^{q} d \mu+D \int|f|^{q} d \mu,
$$

with functions $U$ possessing suitable growth properties at infinity. Our key result in this paper is obtaining (in Sect. 3) for a probability measure $d \mu=\frac{e^{-g(N)}}{Z} d \lambda$, defined with $g(N)$ satisfying suitable growth conditions, the following U-Bound in the setting of the higher-dimensional anisotropic Heisenberg group:

$$
\int \frac{g^{\prime}(N)}{N^{2}}|f|^{q} d \mu \leq C \int|\nabla f|^{q} d \mu+D \int|f|^{q} d \mu,
$$

where $\nabla:=\left(X_{i}\right)_{1 \leq i \leq n}$, with constants $C, D \in(0, \infty)$ independent of the function $f$ for which the right-hand side is well defined. This U-bound is used (in Sect. 4) to get a qPoincaré inequality and a $\beta$-Logarithmic Sobolev inequality for $q \geq 2$. We expect that our results can be used to extend those coercive inequalities to an infinite dimensional setting, which is of interest. (See also works: [5,6,17,29,31,37].) In the second section, we will start by extending Balogh and Tyson's anisotropic Heisenberg group in $\mathbb{R}^{5}$ [2] to a higher-dimensional anisotropic Heisenberg group in $\mathbb{R}^{2 n+1}$, and use Beals et al.'s [3] explicit integral representation to compute the fundamental solution. We also compute bounds for $|\nabla N|$ and $x \cdot \nabla N$ (Sect. 2), which are essential to get the U-Bound (1.3) (Sect. 3). We remark that in our setting, unlike in the case studied in [8], $x \cdot \nabla N$ can be negative, and we will need the dimension $n>5$ to take care of the negative term. For $n \leq 5$, some other method is yet to be explored to get U-Bounds. In the fourth section, we apply the U-Bound to get coercive inequalities like the q-Poincaré inequality and the $\beta$-Logarithmic Sobolev inequality for $q \geq 2$. The fifth section is the "Appendix", where we prove the theorems of Sect. 2.

\section{The fundamental solution}

Let $\theta>0$, and consider a generalisation to the anisotropic Heisenberg group $\mathbb{H}_{2 n}\left(\frac{\theta}{2}, \theta\right)$, as introduced in [2], on $\mathbb{R}^{2 n+1}$ with the dilation $\delta_{\lambda}\left(x_{1}, x_{2}, \ldots, x_{2 n}, t\right)=$ $\left(\lambda x_{1}, \lambda x_{2}, \ldots, \lambda x_{2 n}, \lambda^{2} t\right)$ and the composition law 


$$
\begin{aligned}
& \left(x_{1}, x_{2}, \ldots, x_{2 n}, t\right) \circ\left(\eta_{1}, \eta_{2}, \ldots, \eta_{2 n}, \tau\right) \\
& =\left(x_{1}+\eta_{1}, x_{2}+\eta_{2}, \ldots, x_{2 n}+\eta_{2 n}, t+\tau+\frac{\theta x_{1} \eta_{n+1}}{2}-\frac{\theta x_{n+1} \eta_{1}}{2}\right. \\
& \left.\quad+\theta \sum_{j=2}^{n}\left(x_{j} \eta_{j+n}-\eta_{j} x_{j+n}\right)\right) .
\end{aligned}
$$

$\mathbb{H}_{2 n}\left(\frac{\theta}{2}, \theta\right)$ is a homogeneous Carnot group of step two with generators

$$
X_{j}= \begin{cases}\partial_{x_{1}}-\frac{\theta x_{n+1}}{2} \partial_{t} & j=1 \\ \partial_{x_{n+1}}+\frac{\theta x_{1}}{2} \partial_{t} & j=n+1 \\ \partial_{x_{j}}-\theta x_{j+n} \partial_{t} & j=2,3, \ldots, n \\ \partial_{x_{j}}+\theta x_{j-n} \partial_{t} & j=n+2, n+3, \ldots, 2 n\end{cases}
$$

Theorem 1 The fundamental solution for the group $\mathbb{H}_{2 n}\left(\frac{\theta}{2}, \theta\right)$ is given by the following homogeneous norm

$$
N(x, t)=\frac{\left(B^{2}+t^{2}\right)^{\frac{1}{4 n}}\left(A B+t^{2}+A \sqrt{B^{2}+t^{2}}\right)^{\frac{1}{2}-\frac{1}{4 n}}}{\left(B+\sqrt{B^{2}+t^{2}}\right)^{\frac{1}{2}}}
$$

where $A=\frac{\theta x_{1}^{2}}{2}+\frac{\theta x_{n+1}^{2}}{2}+\frac{\theta}{2} \sum_{j=2, j \neq n+1}^{2 n} x_{j}^{2}$ and $B=\frac{\theta x_{1}^{2}}{4}+\frac{\theta x_{n+1}^{2}}{4}+\frac{\theta}{2} \sum_{j=2, j \neq n+1}^{2 n} x_{j}^{2}$.

Remark Note that for $n=2$ and $\theta=2$, and by using

$$
\left(A B+t^{2}+A \sqrt{B^{2}+t^{2}}\right)=\left(B+\sqrt{B^{2}+t^{2}}\right)\left(A-B+\sqrt{B^{2}+t^{2}}\right),
$$

we get back the fundamental solution as calculated in [2].

For what follows, we denote $|x|=\left(\sum_{j=1}^{2 n} x_{j}^{2}\right)^{\frac{1}{2}}$ to be the Euclidean norm. The following lemma is crucial in obtaining a U-Bound in Sect. 3.

Lemma 2 The homogeneous norm $N$ on $\mathbb{H}_{2 n}\left(\frac{1}{2}, 1\right)$ satisfies

$$
\begin{aligned}
& x \cdot \nabla N \geq-\frac{\theta|x|^{2}}{4 n N}, \\
& |\nabla N|^{2} \geq \frac{\theta^{2}|x|^{2}}{2^{5+\frac{2}{n}} N^{2}},
\end{aligned}
$$

and

$$
|\nabla N|^{2} \leq \frac{\theta^{2}(2 n+1)^{2}|x|^{2}}{2^{3} n^{2} N^{2}}
$$


In the appendix (Sect. 1), we prove Theorem 1 and Lemma 2, which involve lengthy calculations based on a general formula for Green functions for type-2 Carnot groups [3].

\section{U-Bound}

The following U-Bound will be used in Sect. 4 to prove the $q$-Poincaré inequality for $q \geq 2$ for the measure

$$
d \mu=\frac{e^{-g(N)}}{Z} d \lambda
$$

under the condition that $\frac{g^{\prime}(N)}{N^{2}}$ is an increasing function on the anisotropic Heisenberg group where $n>5$. In addition, in Sect. 4, we will prove a $\beta$-Logarithmic Sobolev inequality for $d \mu=\frac{e^{-\alpha N^{p}}}{Z} d \lambda$, for $p \geq 4, q \geq 2$, and $0<\beta \leq \frac{p-3}{p}$.

Theorem 3 Let $N^{-2 n}$ be the fundamental solution in the setting of the anisotropic Heisenberg group $\mathbb{R}^{2 n+1}$ with $n>5$. Let $g:[0, \infty) \rightarrow[0, \infty)$ be a differentiable increasing function such that $g^{\prime \prime}(N) \leq g^{\prime}(N)^{2}$ on $\{N \geq 1\}$. Let $d \mu=\frac{e^{-g(N)}}{Z} d \lambda$ be a probability measure and $Z$ the normalization constant. Then, for $q \geq 2$,

$$
\int \frac{g^{\prime}(N)}{N^{2}}|f|^{q} d \mu \leq C \int|\nabla f|^{q} d \mu+D \int|f|^{q} d \mu
$$

holds outside the unit ball $\{N<1\}$ with $C$ and $D$ positive constants independent of a function $f$ for which the right hand side is well defined.

Proof First, we prove the result for $q=2$ :

Using integration by parts,

$$
\begin{aligned}
\int(\nabla N) \cdot(\nabla f) e^{-g(N)} d \lambda= & -\int \nabla\left(\nabla N e^{-g(N)}\right) f d \lambda=-\int \Delta N f e^{-g(N)} d \lambda \\
& +\int|\nabla N|^{2} f g^{\prime}(N) e^{-g(N)} d \lambda .
\end{aligned}
$$

Since $N^{-2 n}$ is the fundamental solution, we have

$$
\Delta N=|\nabla N|^{2} \frac{(Q-1)}{N}
$$

Hence,

$$
\int|\nabla N|^{2} f\left[g^{\prime}(N)-\frac{(Q-1)}{N}\right] e^{-g(N)} d \lambda=\int(\nabla N) \cdot(\nabla f) e^{-g(N)} d \lambda,
$$


Replacing $f$ by $\frac{f^{2}}{|x|^{2}}$ and using (2.3), the left-hand side of (3.1) becomes:

$$
\begin{aligned}
\int|\nabla N|^{2} \frac{f^{2}}{|x|^{2}}\left[g^{\prime}(N)-\frac{(Q-1)}{N}\right] e^{-g(N)} d \lambda & \geq \frac{\theta^{2}}{2^{5+\frac{2}{n}}} \int f^{2}\left[\frac{g^{\prime}(N)}{N^{2}}-\frac{(Q-1)}{N^{3}}\right] e^{-g(N)} d \lambda \\
& \geq \frac{\theta^{2}}{2^{5+\frac{2}{n}}} \int f^{2}\left[\frac{g^{\prime}(N)}{N^{2}}-(Q-1)\right] e^{-g(N)} d \lambda .
\end{aligned}
$$

Where the last inequality is true since $N>1$. As for the right-hand side of (3.1),

$$
\begin{aligned}
\int(\nabla N) \cdot\left(\nabla\left(\frac{f^{2}}{|x|^{2}}\right)\right) e^{-g(N)} d \lambda & =\int(\nabla N) \cdot\left[2 f \frac{\nabla f}{|x|^{2}}-\frac{2 f^{2} \nabla|x|}{|x|^{3}}\right] e^{-g(N)} d \lambda \\
& =\int(\nabla N) \cdot\left[2 f \frac{\nabla f}{|x|^{2}}-\frac{2 f^{2} x}{|x|^{4}}\right] e^{-g(N)} d \lambda
\end{aligned}
$$

Using the bound on $\nabla N \cdot x$, from (2.2), we get:

$$
\begin{aligned}
\int(\nabla N) \cdot\left(\nabla\left(\frac{f^{2}}{|x|^{2}}\right)\right) e^{-g(N)} d \lambda & =\int \frac{2 f}{|x|^{2}} \nabla N \cdot \nabla f e^{-g(N)} d \lambda-2 \int f^{2} \frac{\nabla N \cdot x}{|x|^{4}} e^{-g(N)} d \lambda \\
& \leq \int \frac{2 f}{|x|^{2}} \nabla N \cdot \nabla f e^{-g(N)} d \lambda+\frac{\theta}{2 n} \int \frac{f^{2}}{N|x|^{2}} e^{-g(N)} d \lambda \\
& \leq 2 \int \frac{f}{|x|^{2}}|\nabla N||\nabla f| e^{-g(N)} d \lambda+\frac{\theta}{2 n} \int \frac{f^{2}}{N|x|^{2}} e^{-g(N)} d \lambda
\end{aligned}
$$

using the bound on $|\nabla N|$, from (2.4), we get

$$
\leq \frac{(2 n+1) \theta}{2^{\frac{1}{2}} n} \int \frac{|f|}{N|x|}|\nabla f| e^{-g(N)} d \lambda+\frac{\theta}{2 n} \int \frac{f^{2}}{N|x|^{2}} e^{-g(N)} d \lambda
$$

applying Cauchy's inequality with $\beta: a b \leq \beta a^{2}+\frac{b^{2}}{4 \beta}$ with $a=\frac{|f|}{N|x|} e^{-\frac{g(N)}{2}}$ and $b=\frac{(2 n+1)}{2^{\frac{1}{2}} n}|\nabla f| e^{-\frac{g(N)}{2}}$,

$\leq \beta \int \frac{f^{2}}{N^{2}|x|^{2}} e^{-g(N)} d \lambda+\frac{\theta}{2 n} \int \frac{f^{2}}{N|x|^{2}} e^{-g(N)} d \lambda+\frac{(2 n+1)^{2} \theta^{2}}{8 n^{2} \beta} \int|\nabla f|^{2} e^{-g(N)} d \lambda$.

Combining the last inequality with (3.2), we get

$$
\begin{aligned}
\frac{\theta^{2}}{2^{5+\frac{2}{n}}} \int f^{2}\left[\frac{g^{\prime}(N)}{N^{2}}-(Q-1)\right] e^{-g(N)} d \lambda \leq & \beta \int \frac{f^{2}}{N^{2}|x|^{2}} e^{-g(N)} d \lambda+\frac{\theta}{2 n} \int \frac{f^{2}}{N|x|^{2}} e^{-g(N)} d \lambda \\
& +\frac{(2 n+1)^{2} \theta^{2}}{8 n^{2} \beta} \int|\nabla f|^{2} e^{-g(N)} d \lambda
\end{aligned}
$$


For $n$ large enough, to be determined later, we need to bound $\frac{\theta}{2 n} \int \frac{f^{2}}{N|x|^{2}} e^{-g(N)} d \lambda$ from the right hand side of (3.4) by $\frac{\theta^{2}}{2^{5+\frac{2}{n}}} \int f^{2} \frac{g^{\prime}(N)}{N^{2}} e^{-g(N)} d \lambda$ by using Hardy's inequality (see [32] and references therein) and the Coarea formula (page 468 of [7]). Since $\beta$ could be chosen to be arbitrarily small and since $N>1$, we do not worry about the term $\beta \int \frac{f^{2}}{N^{2}|x|^{2}} e^{-g(N)} d \lambda$ since it follows the same procedure as $\frac{\theta}{2 n} \int \frac{f^{2}}{N|x|^{2}} e^{-g(N)} d \lambda$. Let $E=\left\{(x, z): \frac{1}{|x|^{2}} \leq \alpha \frac{g^{\prime}(N)}{N}\right\}$ and $F=\left\{(x, z): \alpha \frac{|x|^{2} g^{\prime}(N)}{N}<1\right\}$.

$$
\begin{aligned}
\frac{\theta}{2 n} \int \frac{f^{2}}{N|x|^{2}} e^{-g(N)} d \lambda & =\frac{\theta}{2 n} \int_{F} \frac{f^{2}}{N|x|^{2}} e^{-g(N)} d \lambda+\frac{\theta}{2 n} \int_{E} \frac{f^{2}}{N|x|^{2}} e^{-g(N)} d \lambda \\
& \leq \frac{\theta}{2 n} \int_{F} \frac{f^{2}}{N|x|^{2}} e^{-g(N)} d \lambda+\frac{\alpha \theta}{2 n} \int_{E} \frac{f^{2} g^{\prime}(N)}{N^{2}} e^{-g(N)} d \lambda .
\end{aligned}
$$

where (3.5) is true since $E=\left\{(x, z): \frac{1}{|x|^{2}} \leq \alpha \frac{g^{\prime}(N)}{N}\right\} . \alpha$ is to be chosen later. The aim now is to estimate the first term of (3.5). Consider $F_{r}=\left\{\alpha \frac{|x|^{2} g^{\prime}(N)}{N}<r\right\}$, where $1<r<2$. Integrating by parts:

$$
\begin{aligned}
& \frac{\theta}{2 n} \int_{F_{r}} \frac{\left|f e^{\frac{-g(N)}{2}}\right|^{2}}{N|x|^{2}} d \lambda=\left(\frac{\theta}{2 n}\right) \frac{1}{2 n-2} \int_{F_{r}} \frac{\left|f e^{\frac{-g(N)}{2}}\right|^{2}}{N^{2}} \nabla\left(\frac{x}{|x|^{2}}\right) d \lambda \\
& =-\left(\frac{\theta}{2 n}\right) \frac{1}{2 n-2} \int_{F_{r}} \nabla\left(\left|\frac{f e^{\frac{-g(N)}{2}}}{N^{\frac{1}{2}}}\right|^{2}\right) \cdot \frac{x}{|x|^{2}} d \lambda \\
& +\left(\frac{\theta}{2 n}\right) \frac{1}{2 n-2} \int_{\partial F_{r}} \frac{f^{2} e^{-g(N)}}{N|x|^{2}} \sum_{j=1}^{2 n} \frac{x_{j}<X_{j} I, \nabla_{e u c}\left(\alpha \frac{|x|^{2} g^{\prime}(N)}{N}\right)>}{\left|\nabla_{\text {euc }}\left(\alpha \frac{|x|^{2} g^{\prime}(N)}{N}\right)\right|} d H^{2 n} \\
& =-\left(\frac{\theta}{2 n}\right) \frac{1}{n-1} \int_{F_{r}} \frac{f e^{\frac{-g(N)}{2}}}{N^{\frac{1}{2}}} \nabla\left(\frac{f e^{\frac{-g(N)}{2}}}{N^{\frac{1}{2}}}\right) \cdot \frac{x}{|x|^{2}} d \lambda \\
& +\left(\frac{\theta}{2 n}\right) \frac{1}{2 n-2} \int_{\partial F_{r}} \frac{f^{2} e^{-g(N)}}{N|x|^{2}} \sum_{j=1}^{2 n} \frac{x_{j}<X_{j} I, \nabla_{e u c}\left(\alpha \frac{|x|^{2} g^{\prime}(N)}{N}\right)>}{\left|\nabla_{e u c}\left(\alpha \frac{|x|^{2} g^{\prime}(N)}{N}\right)\right|} d H^{2 n} \\
& \leq \frac{\theta}{4 n} \int_{F_{r}} \frac{\left|f e^{\frac{-g(N)}{2}}\right|^{2}}{N|x|^{2}} d \lambda+\frac{\theta}{4 n(n-1)^{2}} \int_{F_{r}}\left|\nabla\left(\frac{f e^{\frac{-g(N)}{2}}}{N^{\frac{1}{2}}}\right)\right|^{2} d \lambda \\
& +\frac{\theta}{4 n(n-1)} \int_{\partial F_{r}} \frac{f^{2} e^{-g(N)}}{N|x|^{2}} \sum_{j=1}^{2 n} \frac{x_{j}<X_{j} I, \nabla_{\text {euc }}\left(\alpha \frac{|x|^{2} g^{\prime}(N)}{N}\right)>}{\left|\nabla_{\text {euc }}\left(\alpha \frac{|x|^{2} g^{\prime}(N)}{N}\right)\right|} d H^{2 n}
\end{aligned}
$$


Where the last step uses Cauchy's inequality. Subtracting on both sides of the last inequality by $\frac{\theta}{4 n} \int_{F_{r}} \frac{\left|f e^{\frac{-g(N)}{2}}\right|^{2}}{N|x|^{2}} d \lambda$, and using the fact that $1<r<2$, we get:

$$
\begin{aligned}
\left(\frac{\theta}{2 n}\right) \int_{F_{1}} \frac{\left|f e^{\frac{-g(N)}{2}}\right|^{2}}{N|x|^{2}} d \lambda \leq & \left(\frac{\theta}{2 n}\right) \int_{F_{r}} \frac{\left|f e^{\frac{-g(N)}{2}}\right|^{2}}{N|x|^{2}} d \lambda \\
\leq & \left(\frac{\theta}{2 n}\right) \frac{1}{(n-1)^{2}} \int_{F_{r}}\left|\nabla\left(\frac{f e^{\frac{-g(N)}{2}}}{N^{\frac{1}{2}}}\right)\right|^{2} d \lambda \\
& +\left(\frac{\theta}{2 n}\right) \frac{1}{n-1} \int_{\partial F_{r}} \frac{f^{2} e^{-g(N)}}{N|x|^{2}} \sum_{j=1}^{2 n} \frac{x_{j}<X_{j} I, \nabla_{\text {euc }}\left(\alpha \frac{|x|^{2} g^{\prime}(N)}{N}\right)>}{\left|\nabla_{\text {euc }}\left(\alpha \frac{|x|^{2} g^{\prime}(N)}{N}\right)\right|} d H^{2 n} \\
\leq & \left(\frac{\theta}{2 n}\right) \frac{1}{(n-1)^{2}} \int_{F_{2}}\left|\nabla\left(\frac{f e^{\frac{-g(N)}{2}}}{N^{\frac{1}{2}}}\right)\right|^{2} d \lambda \\
& +\left(\frac{\theta}{2 n}\right) \frac{1}{n-1} \int_{\partial F_{r}} \frac{f^{2} e^{-g(N)}}{N|x|^{2}} \sum_{j=1}^{2 n} \frac{x_{j}<X_{j} I, \nabla_{\text {euc }}\left(\alpha \frac{|x|^{2} g^{\prime}(N)}{N}\right)>}{\left|\nabla_{\text {euc }}\left(\alpha \frac{|x|^{2} g^{\prime}(N)}{N}\right)\right|} d H^{2 n}
\end{aligned}
$$

Integrating both sides of the inequality from $r=1$ to $r=2$, we get:

$$
\begin{aligned}
& \left(\frac{\theta}{2 n}\right) \int_{1}^{2} \int_{F_{1}} \frac{\left|f e^{\frac{-g(N)}{2}}\right|^{2}}{N|x|^{2}} d \lambda d r \\
& \leq\left(\frac{\theta}{2 n}\right) \frac{1}{(n-1)^{2}} \int_{1}^{2} \int_{F_{2}}\left|\nabla\left(\frac{f e^{\frac{-g(N)}{2}}}{N^{\frac{1}{2}}}\right)\right|^{2} d \lambda d r \\
& \quad+\left(\frac{\theta}{2 n}\right) \frac{1}{n-1} \int_{1}^{2} \int_{\partial F_{r}} \frac{f^{2} e^{-g(N)}}{N|x|^{2}} \sum_{j=1}^{2 n} \frac{x_{j}<X_{j} I, \nabla_{\text {euc }}\left(\alpha \frac{|x|^{2} g^{\prime}(N)}{N}\right)>}{\left|\nabla_{\text {euc }}\left(\alpha \frac{|x|^{2} g^{\prime}(N)}{N}\right)\right|} d H^{2 n} d r
\end{aligned}
$$

To recover the full measure in the boundary term, we use the Coarea formula:

$$
\begin{aligned}
& \left(\frac{\theta}{2 n}\right) \int_{F_{1}} \frac{\left|f e^{\frac{-g(N)}{2}}\right|^{2}}{N|x|^{2}} d \lambda \leq \frac{\theta}{2 n(n-1)^{2}} \int_{F_{2}}\left|\nabla\left(\frac{f e^{\frac{-g(N)}{2}}}{N^{\frac{1}{2}}}\right)\right|^{2} d \lambda \\
& +\frac{\theta}{2 n(n-1)} \int_{\left\{1<\alpha \frac{|x|^{2} g^{\prime}(N)}{N}<2\right\}} \frac{f^{2} e^{-g(N)}}{N|x|^{2}} \sum_{j=1}^{2 n} x_{j}<X_{j} I, \nabla_{\text {euc }}\left(\alpha \frac{|x|^{2} g^{\prime}(N)}{N}\right)>d \lambda
\end{aligned}
$$

It remains to compute the right hand side of (3.6). The first term,

$$
\begin{aligned}
A & =\left(\frac{\theta}{2 n}\right) \frac{1}{(n-1)^{2}} \int_{F_{2}}\left|\nabla\left(\frac{f e^{\frac{-g(N)}{2}}}{N^{\frac{1}{2}}}\right)\right|^{2} d \lambda \\
& =\left(\frac{\theta}{2 n}\right) \frac{1}{(n-1)^{2}} \int_{F_{2}}\left|\nabla f \frac{e^{-\frac{g(N)}{2}}}{N^{\frac{1}{2}}}-f \frac{g^{\prime}(N)}{2} \frac{\nabla N e^{-\frac{g(N)}{2}}}{N^{\frac{1}{2}}}-\frac{1}{2} \frac{f \nabla N e^{-\frac{g(N)}{2}}}{N^{\frac{3}{2}}}\right|^{2} d \lambda \\
& =\left(\frac{\theta}{2 n}\right) \frac{1}{(n-1)^{2}} \int_{F_{2}} \frac{|\nabla f|^{2}}{N} e^{-g(N)} d \lambda+\left(\frac{\theta}{8 n}\right) \frac{1}{(n-1)^{2}} \int_{F_{2}} f^{2} g^{\prime}(N)^{2} \frac{|\nabla N|^{2}}{N} e^{-g(N)} d \lambda
\end{aligned}
$$




$$
\begin{aligned}
& +\left(\frac{\theta}{8 n}\right) \frac{1}{(n-1)^{2}} \int_{F_{2}} f^{2} \frac{|\nabla N|^{2}}{N^{3}} e^{-g(N)} d \lambda-\frac{\theta}{2 n(n-1)^{2}} \int_{F_{2}} f \nabla f \cdot \nabla N \frac{g^{\prime}(N)}{N} e^{-g(N)} d \lambda \\
& -\frac{\theta}{2 n(n-1)^{2}} \int_{F_{2}} f \nabla f \cdot \nabla N \frac{1}{N^{2}} e^{-g(N)} d \lambda+\frac{\theta}{4 n(n-1)^{2}} \int_{F_{2}} f^{2} g^{\prime}(N) \frac{|\nabla N|^{2}}{N^{2}} e^{-g(N)} d \lambda .
\end{aligned}
$$

Using (2.4),

$$
\begin{aligned}
A \leq & \left(\frac{\theta}{2 n}\right) \frac{1}{(n-1)^{2}} \int_{F_{2}} \frac{|\nabla f|^{2}}{N} e^{-g(N)} d \lambda+\frac{(2 n+1)^{2} \theta^{3}}{2^{6} n^{3}(n-1)^{2}} \int_{F_{2}} f^{2} g^{\prime}(N)^{2} \frac{|x|^{2}}{N^{3}} e^{-g(N)} d \lambda \\
& +\frac{(2 n+1)^{2} \theta^{3}}{2^{6} n^{3}(n-1)^{2}} \int_{F_{2}} f^{2} \frac{|x|^{2}}{N^{5}} e^{-g(N)} d \lambda+\frac{(2 n+1) \theta^{2}}{2^{\frac{5}{2}} n^{2}(n-1)^{2}} \int_{F_{2}}|f||\nabla f| \frac{g^{\prime}(N)|x|}{N^{2}} e^{-g(N)} d \lambda \\
& +\frac{(2 n+1) \theta^{2}}{2^{\frac{5}{2}} n^{2}(n-1)^{2}} \int_{F_{2}}|f||\nabla f| \frac{|x|}{N^{3}} e^{-g(N)} d \lambda+\frac{(2 n+1)^{2} \theta^{3}}{2^{5} n^{3}(n-1)^{2}} \int_{F_{2}} f^{2} g^{\prime}(N) \frac{|x|^{2}}{N^{4}} e^{-g(N)} d \lambda .
\end{aligned}
$$

Using the fact that $F_{2}=\left\{\alpha \frac{|x|^{2} g^{\prime}(N)}{N}<2\right\}$ and that $|x| \leq C_{n} N$,

$$
\begin{aligned}
A \leq & \left(\frac{1}{2 n}\right) \frac{\theta}{(n-1)^{2}} \int_{F_{2}} \frac{|\nabla f|^{2}}{N} e^{-g(N)} d \lambda+\frac{(2 n+1)^{2} \theta^{3}}{\alpha 2^{5} n^{3}(n-1)^{2}} \int_{F_{2}} f^{2} \frac{g^{\prime}(N)}{N^{2}} e^{-g(N)} d \lambda \\
& +\frac{(2 n+1)^{2} \theta^{3} C_{n}^{2}}{2^{6} n^{3}(n-1)^{2}} \int_{F_{2}} f^{2} \frac{1}{N^{3}} e^{-g(N)} d \lambda+\frac{(2 n+1) \theta^{2} C_{n}}{2^{\frac{5}{2}} n^{2}(n-1)^{2}} \int_{F_{2}}|f||\nabla f| \frac{g^{\prime}(N)}{N} e^{-g(N)} d \lambda \\
& +\frac{(2 n+1) \theta^{2} C_{n}}{2^{\frac{5}{2}} n^{2}(n-1)^{2}} \int_{F_{2}}|f||\nabla f| \frac{1}{N^{2}} e^{-g(N)} d \lambda+\frac{(2 n+1)^{2} \theta^{3}}{\alpha 2^{4} n^{3}(n-1)^{2}} \int_{F_{2}} f^{2} \frac{1}{N^{3}} e^{-g(N)} d \lambda .
\end{aligned}
$$

Using Cauchy's inequality with $\gamma: a b \leq \gamma a^{2}+\frac{b^{2}}{4 \gamma}$ on $\frac{(2 n+1) \theta^{2} C_{n}}{2^{\frac{5}{2}} n^{2}(n-1)^{2}} \int_{F_{2}}|f||\nabla f| \frac{g^{\prime}(N)}{N}$ $e^{-g(N)} d \lambda$ with $a=\frac{|f| g^{\prime}(N)}{N}$ and $b=\frac{(2 n+1) \theta^{2} C_{n}}{2^{\frac{5}{2}} n^{2}(n-1)^{2}}|\nabla f|$ and Cauchy's inequality with $\gamma$ on $\frac{(2 n+1) \theta^{2} C_{n}}{2^{\frac{5}{2}} n^{2}(n-1)^{2}} \int_{F_{2}}|f||\nabla f| \frac{1}{N^{2}} e^{-g(N)} d \lambda$ with $a=\frac{|f|}{N^{2}}$ and $b=\frac{(2 n+1) \theta^{2} C_{n}}{2^{\frac{5}{2}} n^{2}(n-1)^{2}}|\nabla f|$ in addition to $N>1$,

$$
\begin{aligned}
A \leq & \left(\left(\frac{\theta}{2 n}\right) \frac{1}{(n-1)^{2}}+\frac{\theta^{4} C_{n}^{2}(2 n+1)^{2}}{\gamma 2^{6} n^{4}(n-1)^{4}}\right) \int_{F_{2}}|\nabla f|^{2} e^{-g(N)} d \lambda \\
& +\left(\frac{\theta^{3} C_{n}^{2}(2 n+1)^{2}}{2^{6} n^{3}(n-1)^{2}}+\frac{\theta^{3}(2 n+1)^{2}}{\alpha 2^{4} n^{3}(n-1)^{2}}+\gamma\right) \int_{F_{2}} f^{2} e^{-g(N)} d \lambda \\
& ++\left(\frac{\theta^{3}(2 n+1)^{2}}{\alpha 2^{5} n^{3}(n-1)^{2}}+\gamma\right) \int_{F_{2}} f^{2} \frac{g^{\prime}(N)}{N^{2}} e^{-g(N)} d \lambda .
\end{aligned}
$$

Hence,

$$
A \leq\left(\frac{\theta^{3}(2 n+1)^{2}}{\alpha 2^{5} n^{3}(n-1)^{2}}+\gamma\right) \int_{F_{2}} f^{2} \frac{g^{\prime}(N)}{N^{2}} e^{-g(N)} d \lambda+C \int_{F_{2}}|\nabla f|^{2} e^{-g(N)} d \lambda+D \int_{F_{2}} f^{2} e^{-g(N)} d \lambda
$$


For the second term of (3.6),

$$
B=\left(\frac{\theta}{2 n}\right) \frac{1}{n-1} \int_{\left\{1<\alpha \frac{|x|^{2} g^{\prime}(N)}{N}<2\right\}} \frac{f^{2} e^{-g(N)}}{N|x|^{2}} \sum_{j=1}^{2 n} x_{j}<X_{j} I, \nabla_{\text {euc }}\left(\alpha \frac{|x|^{2} g^{\prime}(N)}{N}\right)>d \lambda .
$$

On the anisotropic Heisenberg group, for $e_{i}$ the standard Euclidean basis on $\mathbb{R}^{2 n+1}$,

$$
X_{j} I \cdot e_{i}= \begin{cases}0 & \text { for } i \neq j \text { and } i \leq 2 n \\ 1 & \text { for } i=j \text { and } i \leq 2 n \\ \sum_{l=1}^{2 n} \Lambda_{j l} x_{l} & \text { for } i=2 n+1\end{cases}
$$

where

$$
\Lambda_{j l}= \begin{cases}-\frac{\theta}{2} & j=1, \quad l=n+1 \\ \frac{\theta}{2} & j=n+1, \quad l=1 \\ -\theta & j=2,3, \ldots, n, \quad l=k+n \\ \theta & j=n+2, n+3, \ldots, 2 n, \quad l=k-n \\ 0 & \text { otherwise }\end{cases}
$$

Also,

$$
\nabla_{\text {euc }}\left(\alpha \frac{|x|^{2} g^{\prime}(N)}{N}\right) \cdot e_{i}= \begin{cases}2 \alpha \frac{x_{i} g^{\prime}(N)}{N}+\frac{\alpha|x|^{2} g^{\prime \prime}(N) \partial_{x_{i}} N}{N}-\frac{\alpha|x|^{2} g^{\prime}(N) \partial_{x_{i}} N}{N^{2}} & \text { for } i=j \text { and } i \leq 2 n \\ \frac{\alpha|x|^{2} g^{\prime \prime}(N) \partial_{t} N}{N}-\frac{\alpha|x|^{2} g^{\prime}(N) \partial_{t} N}{N^{2}} & \text { for } i=2 n+1 .\end{cases}
$$

Taking the dot product and summing,

$$
\begin{aligned}
& \sum_{j=1}^{2 n} x_{j}<X_{j} I, \nabla_{e u c}\left(\alpha \frac{|x|^{2} g^{\prime}(N)}{N}\right)>=2 \alpha \frac{|x|^{2} g^{\prime}(N)}{N}+\sum_{j=1}^{2 n} x_{j} \frac{\alpha|x|^{2} g^{\prime \prime}(N) \partial_{x_{j}} N}{N} \\
& -\sum_{j=1}^{2 n} x_{j} \frac{\alpha|x|^{2} g^{\prime}(N) \partial_{x_{j}} N}{N^{2}}+\sum_{j=1}^{2 n} x_{j}\left(\frac{\alpha|x|^{2} g^{\prime \prime}(N) \partial_{t} N}{N}-\frac{\alpha|x|^{2} g^{\prime}(N) \partial_{t} N}{N^{2}}\right) \sum_{l=1}^{2 n} \Lambda_{j l} x_{l} \\
& =2 \alpha \frac{|x|^{2} g^{\prime}(N)}{N}+\frac{\alpha|x|^{2} g^{\prime \prime}(N) x \cdot \nabla N}{N}-\frac{\alpha|x|^{2} g^{\prime}(N) x \cdot \nabla N}{N^{2}} \\
& \quad+\left(\frac{\alpha|x|^{2} g^{\prime \prime}(N) \partial_{t} N}{N}-\frac{\alpha|x|^{2} g^{\prime}(N) \partial_{t} N}{N^{2}}\right) \sum_{j=1}^{2 n} \sum_{l=1}^{2 n} \Lambda_{j l} x_{l} x_{j} \\
& =2 \alpha \frac{|x|^{2} g^{\prime}(N)}{N}+\frac{\alpha|x|^{2} g^{\prime \prime}(N) x \cdot \nabla N}{N}-\frac{\alpha|x|^{2} g^{\prime}(N) x \cdot \nabla N}{N^{2}}
\end{aligned}
$$

where $\sum_{j=1}^{2 n} \sum_{l=1}^{2 n} \Lambda_{j l} x_{l} x_{j}=0$ since $\Lambda$ is skew symmetric. Hence, using (2.4),

$$
\left|\sum_{j=1}^{2 n} x_{j}<X_{j} I, \nabla_{\text {euc }}\left(\alpha \frac{|x|^{2} g^{\prime}(N)}{N}\right)>\right| \leq 2 \alpha \frac{|x|^{2} g^{\prime}(N)}{N}+\frac{\alpha|x|^{3} g^{\prime \prime}(N)|\nabla N|}{N}+\frac{\alpha|x|^{3} g^{\prime}(N)|\nabla N|}{N^{2}}
$$




$$
\leq 2 \alpha \frac{|x|^{2} g^{\prime}(N)}{N}+\frac{\alpha \theta(2 n+1)|x|^{4} g^{\prime \prime}(N)}{2^{\frac{3}{2}} n N^{2}}+\frac{\alpha \theta(2 n+1)|x|^{4} g^{\prime}(N)}{2^{\frac{3}{2}} n N^{3}} .
$$

Therefore, replacing,

$$
\begin{aligned}
B=\left(\frac{\theta}{2 n}\right) & \frac{1}{n-1} \int_{\left\{1<\alpha \frac{|x|^{2} g^{\prime}(N)}{N}<2\right\}} \frac{f^{2} e^{-g(N)}}{N|x|^{2}} \sum_{j=1}^{2 n} x_{j}<X_{j} I, \nabla_{\text {euc }}\left(\alpha \frac{|x|^{2} g^{\prime}(N)}{N}\right)>d \lambda \\
\leq & \left(\frac{\theta}{n}\right) \frac{\alpha}{n-1} \int_{\left\{1<\alpha \frac{|x|^{2} g^{\prime}(N)}{N}<2\right\}} \frac{f^{2} g^{\prime}(N)}{N^{2}} e^{-g(N)} d \lambda \\
& +\frac{\theta^{2} \alpha(2 n+1)}{2^{\frac{5}{2}} n^{2}(n-1)} \int_{\left\{1<\alpha \frac{|x|^{2} g^{\prime}(N)}{N}<2\right\}} \frac{f^{2}|x|^{2} g^{\prime \prime}(N)}{N^{3}} e^{-g(N)} d \lambda \\
& +\frac{\theta^{2} \alpha(2 n+1)}{2^{\frac{5}{2}} n^{2}(n-1)} \int_{\left\{1<\alpha \frac{|x|^{2} g^{\prime}(N)}{N}<2\right\}} \frac{f^{2}|x|^{2} g^{\prime}(N)}{N^{4}} e^{-g(N)} d \lambda .
\end{aligned}
$$

Using the fact that we are integrating over $\left\{1<\alpha \frac{|x|^{2} g^{\prime}(N)}{N}<2\right\}$,

$$
\begin{aligned}
B \leq & \left(\frac{\theta}{n}\right) \frac{\alpha}{n-1} \int_{\left\{1<\alpha \frac{|x|^{2} g^{\prime}(N)}{N}<2\right\}} \frac{f^{2} g^{\prime}(N)}{N^{2}} e^{-g(N)} d \lambda+\frac{(2 n+1) \theta^{2}}{2^{\frac{3}{2}} n^{2}(n-1)} \int_{\left\{1<\alpha \frac{|x|^{2} g^{\prime}(N)}{N}<2\right\}} \frac{f^{2} g^{\prime \prime}(N)}{N^{2} g^{\prime}(N)} e^{-g(N)} d \lambda \\
& +\frac{\theta^{2}(2 n+1)}{2^{\frac{3}{2}} n^{2}(n-1)} \int_{\left\{1<\alpha \frac{|x|^{2} g^{\prime}(N)}{N}<2\right\}} \frac{f^{2}}{N^{3}} e^{-g(N)} d \lambda .
\end{aligned}
$$

Using the condition of the theorem that $g^{\prime \prime}(N) \leq g^{\prime}(N)^{2}$ on $\{N \geq 1\}$, we bound the second term on the right hand side of (3.8). Now we go back to (3.4):

$$
\begin{aligned}
& \frac{\theta^{2}}{2^{5+\frac{2}{n}}} \int f^{2}\left[\frac{g^{\prime}(N)}{N^{2}}-(Q-1)\right] e^{-g(N)} d \lambda \\
& \leq \beta \int \frac{f^{2}}{N^{2}|x|^{2}} e^{-g(N)} d \lambda+\frac{\theta}{2 n} \int \frac{f^{2}}{N|x|^{2}} e^{-g(N)} d \lambda+\frac{\theta^{2}(2 n+1)^{2}}{8 n^{2} \beta} \int|\nabla f|^{2} e^{-g(N)} d \lambda .
\end{aligned}
$$

From (3.5) we have that

$$
\frac{\theta}{2 n} \int \frac{f^{2}}{N|x|^{2}} e^{-g(N)} d \lambda \leq \frac{\theta}{2 n} \int_{F} \frac{f^{2}}{N|x|^{2}} e^{-g(N)} d \lambda+\frac{\alpha \theta}{2 n} \int_{E} \frac{f^{2} g^{\prime}(N)}{N^{2}} e^{-g(N)} d \lambda .
$$

From (3.7) and (3.8) we have that

$$
\begin{aligned}
& \frac{\theta}{2 n} \int_{F} \frac{f^{2}}{N|x|^{2}} e^{-g(N)} d \lambda \leq\left(\frac{(2 n+1)^{2} \theta^{3}}{\alpha 2^{5} n^{3}(n-1)^{2}}+\gamma+\frac{\alpha \theta}{n(n-1)}\right) \\
& \int_{F_{2}} f^{2} \frac{g^{\prime}(N)}{N^{2}} e^{-g(N)} d \lambda+C \int_{F_{2}}|\nabla f|^{2} e^{-g(N)} d \lambda+D \int_{F_{2}} f^{2} e^{-g(N)} d \lambda
\end{aligned}
$$

We combine the last three inequalities and repeat the same procedure to $\beta \int \frac{f^{2}}{N^{2}|x|^{2}} e^{-g(N)} d \lambda$ to get:

$$
\left(\frac{\theta^{2}}{2^{5+\frac{2}{n}}}-\frac{\alpha \theta}{2 n}-\frac{(2 n+1)^{2} \theta^{3}}{\alpha 2^{5} n^{3}(n-1)^{2}}-\frac{\alpha \theta}{n(n-1)}-\gamma-\beta\right) \int f^{2} \frac{g^{\prime}(N)}{N^{2}} e^{-g(N)} d \lambda
$$




$$
\leq C \int_{F_{2}}|\nabla f|^{2} e^{-g(N)} d \lambda+D \int_{F_{2}} f^{2} e^{-g(N)} d \lambda .
$$

To get the U-Bound, we need the left hand side of (3.9) to be positive i.e. and find suitable $\alpha, n, \gamma$, and $\beta$. Since $\gamma$ and $\beta$ can be chosen to be arbitrarily small, we need to find solutions to the following inequality:

$$
\frac{\theta}{2^{5+\frac{2}{n}}}-\frac{\alpha}{2 n}-\frac{(2 n+1)^{2} \theta^{2}}{\alpha 2^{5} n^{3}(n-1)^{2}}-\frac{\alpha}{n(n-1)}>0 .
$$

First, we determine $\alpha$ : Let $f(\alpha)=\frac{\theta}{2^{5+\frac{2}{n}}}-\frac{\alpha}{2 n}-\frac{(2 n+1)^{2} \theta^{2}}{\alpha 2^{5} n^{3}(n-1)^{2}}-\frac{\alpha}{n(n-1)}$.

$$
f^{\prime}(\alpha)=-\frac{1}{2 n}+\frac{(2 n+1)^{2} \theta^{2}}{\alpha^{2} 2^{5} n^{3}(n-1)^{2}}-\frac{1}{n(n-1)} .
$$

Solving for $f^{\prime}(\alpha)=0$,

$$
\alpha=\frac{\theta(2 n+1)}{2^{2} n\left(n^{2}-1\right)^{\frac{1}{2}}} .
$$

Replacing $\alpha$ in (3.10),

$$
\begin{aligned}
& \frac{\theta}{2^{5+\frac{2}{n}}}-\frac{\theta(2 n+1)}{2^{3} n^{2}\left(n^{2}-1\right)^{\frac{1}{2}}}-\frac{\theta\left(n^{2}-1\right)^{\frac{1}{2}}(2 n+1)}{2^{3} n^{2}(n-1)^{2}}-\frac{\theta(2 n+1)}{2^{2} n^{2}(n-1)\left(n^{2}-1\right)^{\frac{1}{2}}}>0 \\
& 1>2^{3+\frac{2}{n}}\left(\frac{2 n+1}{n^{2}}\right)\left(\frac{(n+1)^{\frac{1}{2}}}{(n-1)^{\frac{3}{2}}}\right) .
\end{aligned}
$$

This holds true if we choose $n>5$. Hence, we get:

$$
\int f^{2}\left(\frac{g^{\prime}(N)}{N^{2}}\right) e^{-g(N)} d \lambda \leq C \int|\nabla f|^{2} e^{-g(N)} d \lambda+D \int f^{2} e^{-g(N)} d \lambda
$$

Second, for $q>2$, replacing $|f|$ by $|f|^{\frac{q}{2}}$, we get:

$$
\int \frac{g^{\prime}(N)}{N^{2}}|f|^{q} d \mu \leq\left.\left. C \int|\nabla| f\right|^{\frac{q}{2}}\right|^{2} d \mu+D \int|f|^{q} d \mu .
$$

Calculating,

$$
\begin{aligned}
\left.\left.\int|\nabla| f\right|^{\frac{q}{2}}\right|^{2} d \mu & =\left.\left.\int\left|\frac{q}{2}\right| f\right|^{\frac{q-2}{2}}(\operatorname{sgn}(f)) \nabla f\right|^{2} d \mu \\
& \leq \int \frac{q^{2}}{4}|f|^{q-2}|\nabla f|^{2} d \mu .
\end{aligned}
$$


Using Hölder's inequality,

$$
\begin{aligned}
\left.\left.\int|\nabla| f\right|^{\frac{q}{2}}\right|^{2} d \mu & \leq \int \frac{q^{2}}{4}|f|^{q-2}|\nabla f|^{2} d \mu \leq \frac{q^{2}}{4}\left(\int|f|^{q} d \mu\right)^{\frac{q-2}{q}}\left(\int|\nabla f|^{q} d \mu\right)^{\frac{2}{q}} \\
& \leq \frac{q(q-2)}{4} \int|f|^{q} d \mu+\frac{q}{2} \int|\nabla f|^{q} d \mu .
\end{aligned}
$$

where the last inequality uses $a b \leq \frac{a^{p^{\prime}}}{p^{\prime}}+\frac{b^{q^{\prime}}}{q^{\prime}}$, with $a=\left(\int|f|^{q} d \mu\right)^{\frac{q-2}{q}}$,

$b=\left(\int|\nabla f|^{q} d \mu\right)^{\frac{2}{q}}$, and $p^{\prime}$ and $q^{\prime}$ are conjugates. Choosing $p^{\prime}=\frac{q}{q-2}$, we obtain $\frac{1}{q^{\prime}}=1-\frac{q-2}{q}$, so $q^{\prime}=\frac{q}{2}$. Using the inequalities (3.11) and (3.12), we get,

$$
\begin{aligned}
\int \frac{g^{\prime}(N)}{N^{2}}|f|^{q} d \mu & \leq\left.\left. C \int|\nabla| f\right|^{\frac{q}{2}}\right|^{2} d \mu+D \int|f|^{q} d \mu \\
& \leq C^{\prime} \int|\nabla f|^{q} d \mu+D^{\prime} \int|f|^{q} d \mu
\end{aligned}
$$

\section{$4 q$-Poincaré and $\beta$-logarithmic Sobolev inequalities}

We now have the U-Bound (1.3) at our disposal and are ready to prove the q-Poincaré inequality using the U-Bound method of [18]:

Let $\lambda$ be a measure satisfying the q-Poincaré inequality for every ball $B_{R}=\{x: N(x)<R\}$, i.e. there exists a constant $C_{R} \in(0, \infty)$ such that

$$
\frac{1}{\left|B_{R}\right|} \int_{B_{R}}\left|f-\frac{1}{\left|B_{R}\right|} \int_{B_{R}} f\right|^{q} d \lambda \leq C_{R} \frac{1}{\left|B_{R}\right|} \int_{B_{R}}|\nabla f|^{q} d \lambda,
$$

where $1 \leq q<\infty$. Note that we have this Poincaré inequality on balls in the setting of Nilpotent lie groups thanks to Jerison's celebrated paper [21]. Later on, we apply the following result of

Theorem 4 (Hebisch and Zegarliński [18]) Let $\mu$ be a probability measure on $\mathbb{R}^{m}$ which is absolutely continuous with respect to the measure $\lambda$ and such that

$$
\int f^{q} \eta d \mu \leq C \int|\nabla f|^{q} d \mu+D \int f^{q} d \mu
$$

with some non-negative function $\eta$ and some constants $C, D \in(0, \infty)$ independent of a function $f$. Iffor any $L \in(0, \infty)$ there is a constant $A_{L}$ such that $\frac{1}{A_{L}} \leq \frac{d \mu}{d \lambda} \leq A_{L}$ on the set $\{\eta<L\}$ and, for some $R \in(0, \infty)$ (depending on $L$ ), we have $\{\eta<L\} \subset B_{R}$, then $\mu$ satisfies the q-Poincaré inequality

$$
\mu|f-\mu f|^{q} \leq c \mu|\nabla f|^{q}
$$


with some constant $c \in(0, \infty)$ independent of $f$.

The role of $\eta$ in Theorem 4 is played by $\frac{g^{\prime}(N)}{N^{2}}$ from the U-Bound of Theorem 3 . Hence, we get the following corollaries:

Corollary 5 The Poincaré inequality for $q \geq 2$ holds for the measure $d \mu=\frac{\exp \left(-\cosh \left(N^{k}\right)\right)}{Z} d \lambda$, where $\lambda$ is the Lebesgue measure, and $k \geq 1$ in the setting of the anisotropic Heisenberg group $\mathbb{R}^{2 n+1}$ with $n>5$.

Proof $g(N)=\cosh \left(N^{k}\right)$, so $g^{\prime}(N)=k N^{k-1} \sinh \left(N^{k}\right)$, and $g^{\prime \prime}(N)=k(k-1)$ $N^{k-2} \sinh \left(N^{k}\right)+k^{2} N^{2 k-2} \cosh \left(N^{k}\right)$. First $g^{\prime \prime}(N) \leq k^{2}$

$N^{2 k-2} \sinh ^{2}\left(N^{k}\right)=g^{\prime}(N)^{2}$, on the set $\left\{N>\frac{3}{2}\right\}$, so the condition of Theorem 2 is satisfied. Secondly,

$$
\begin{aligned}
& \int \frac{g^{\prime}(N)}{N^{2}} f^{q} d \mu=\int f^{q}\left[k N^{k-3} \sinh \left(N^{k}\right)\right] d \mu \\
& =\int_{\left\{N<\frac{3}{2}\right\}} f^{q}\left[k N^{k-3} \frac{e^{N^{k}}-e^{-N^{k}}}{2}\right] d \mu+\int_{\left\{N \geq \frac{3}{2}\right\}} f^{q}\left[k N^{k-3} \frac{e^{N^{k}}-e^{-N^{k}}}{2}\right] d \mu \\
& \leq \int_{\left\{N<\frac{3}{2}\right\}} f^{q}\left[\left(\frac{3}{2}\right)^{k-3} k \frac{e^{\left(\frac{3}{2}\right)^{k}}-e^{-\left(\frac{3}{2}\right)^{k}}}{2}\right] d \mu+C^{\prime} \int_{\{N \geq 1\}}|\nabla f|^{q} d \mu+D^{\prime} \int_{\{N \geq 1\}}|f|^{q} d \mu \\
& \leq C \int|\nabla f|^{q} d \mu+D \int|f|^{q} d \mu .
\end{aligned}
$$

Thus, the conditions of Theorem 4 are satisfied for $\eta=k N^{k-3} \sinh \left(N^{k}\right)$, and $k \geq 1$. So, the Poincaré inequality holds for $q \geq 2$.

Corollary 6 The Poincaré inequality for $q \geq 1$ holds for the measure $d \mu=\frac{\exp \left(-N^{k}\right)}{Z} d \lambda$, where $\lambda$ is the Lebesgue measure, and $k \geq 4$ in the setting of the anisotropic Heisenberg group $\mathbb{R}^{2 n+1}$ with $n>5$.

Proof Let $g(N)=N^{k}$, so $g^{\prime}(N)=k N^{k-1}$, and $g^{\prime \prime}(N)=k(k-1) N^{k-2}$. First $g^{\prime \prime}(N) \leq k^{2} N^{2 k-2}=g^{\prime}(N)^{2}$, so the condition of Theorem 2 is satisfied. Second,

$$
\begin{aligned}
\int \frac{g^{\prime}(N)}{N^{2}} f^{q} d \mu & =\int f^{q}\left[k N^{k-3}\right] d \mu=\int_{\{N<1\}} f^{q}\left[k N^{k-3}\right] d \mu+\int_{\{N \geq 1\}} f^{q}\left[k N^{k-3}\right] d \mu \\
& \leq \int_{\{N<1\}} k f^{q} d \mu+C^{\prime} \int_{\{N \geq 1\}}|\nabla f|^{q} d \mu+D^{\prime} \int_{\{N \geq 1\}}|f|^{q} d \mu \\
& \leq C \int|\nabla f|^{q} d \mu+D \int|f|^{q} d \mu .
\end{aligned}
$$

Thus, the conditions of Theorem 4 are satisfied for $\eta=k N^{k-3}$, and $k \geq 4$. So, the Poincaré inequality holds for $q \geq 2$.

The following corollary improves Corollary 6 in an interesting way. Namely, at a cost of a logarithmic factor, we now get the Poincaré inequality for polynomial growth of order $k \geq 3$. 
Corollary 7 The Poincaré inequality for $q \geq 2$ holds for the measure $d \mu$ $=\frac{\exp \left(-N^{k} \log (N+1)\right)}{Z} d \lambda$, where $\lambda$ is the Lebesgue measure, and $k \geq 3$ in the setting of the anisotropic Heisenberg group $\mathbb{R}^{2 n+1}$ with $n>5$.

Proof Let $g(N)=N^{k} \log (N+1)$, so $g^{\prime}(N)=k N^{k-1} \log (N+1)+\frac{N^{k}}{N+1}$, and

$$
g^{\prime \prime}(N)=k(k-1) N^{k-2} \log (N+1)+\frac{2 k N^{k-1}}{N+1}-\frac{N^{k}}{(N+1)^{2}} .
$$

First $g^{\prime \prime}(N) \leq k^{2} N^{2 k-2}(\log (N+1))^{2}+\frac{N^{2 k}}{(N+1)^{2}}+2 k N^{k-1} \log (N+1) \frac{N^{k-1}}{N+1}=$ $g^{\prime}(N)^{2}$, so the condition of Theorem 2 is satisfied. Secondly,

$$
\begin{aligned}
\int \frac{g^{\prime}(N)}{N^{2}} f^{q} d \mu= & \int f^{q}\left[k N^{k-3} \log (N+1)+\frac{N^{k-2}}{N+1}\right] d \mu \\
= & \int_{\{N<1\}} f^{q}\left[k N^{k-3} \log (N+1)+\frac{N^{k-2}}{N+1}\right] d \mu \\
& +\int_{\{N \geq 1\}} f^{q}\left[k N^{k-3} \log (N+1)+\frac{N^{k-2}}{N+1}\right] d \mu \\
\leq & \int_{\{N<1\}}(k \log (2)+1) f^{q} d \mu+C^{\prime} \int_{\{N \geq 1\}}|\nabla f|^{q} d \mu+D^{\prime} \int_{\{N \geq 1\}}|f|^{q} d \mu \\
\leq & C \int|\nabla f|^{q} d \mu+D \int|f|^{q} d \mu .
\end{aligned}
$$

Thus, the conditions of Theorem 4 are satisfied for $\eta=k N^{k-3} \log (N+1)+\frac{N^{k-2}}{N+1}$, and $k \geq 3$. So, the Poincaré inequality holds for $q \geq 1$.

To get the $\beta$-Logarithmic Sobolev inequality, we use the following theorem by the authors of this paper in [8] (which generalises Inglis et al.'s Theorem 2.1 [20]).

Theorem 8 Let $U$ be a locally Lipschitz function on $\mathbb{R}^{N}$ which is bounded below such that $Z=\int e^{-U} d \lambda<\infty$, and $d \mu=\frac{e^{-U}}{Z} d \lambda$. Let $\phi:[0, \infty) \rightarrow \mathbb{R}^{+}$be a non-negative, non-decreasing, concave function such that $\phi(0)>0$, and $\phi^{\prime}(0)>0$. Assume the following classical Sobolev inequality is satisfied:

$$
\left(\int|f|^{q+\epsilon} d \lambda\right)^{\frac{q}{q+\epsilon}} \leq a \int|\nabla f|^{q} d \lambda+b \int|f|^{q} d \lambda
$$

for some $a, b \in[0, \infty)$, and $\epsilon>0$. Moreover, if for some $A, B \in[0, \infty)$, we have:

$$
\mu\left(|f|^{q}\left(\phi(U)+|\nabla U|^{q}\right)\right) \leq A \mu|\nabla f|^{q}+B \mu|f|^{q},
$$

Then, there exists constants $C, D \in[0, \infty)$ such that:

$$
\mu\left(|f|^{q} \phi\left(\left|\log \frac{|f|^{q}}{\mu|f|^{q}}\right|\right)\right) \leq C \mu|\nabla f|^{q}+D \mu|f|^{q},
$$


for all locally Lipschitz functions $f$.

Theorem 10 of [8] proves that for $d \mu=\frac{e^{-\alpha N^{p}}}{Z} d \lambda$, where $\alpha>0, p \geq 1, N$ is a smooth homogeneous norm, and $Z$ is the normalization constant, the measure $\mu$ satisfies no $\beta$-logarithmic Sobolev inequality $(0<\beta \leq 1)$ for $1<q<\frac{2 p \beta}{p-1}$. However for $p \geq 4,0<\beta \leq \frac{p-3}{p}$, and $q \geq 2$, we will show that $\mu$ satisfies $\beta$ logarithmic Sobolev inequality.

We will use the following theorem:

Theorem 9 Let $\mathbb{R}^{2 n+1}$ be an anisotropic Heisenberg group with $n>5$. Let the probability measure be $d \mu=\frac{e^{-g(N)}}{Z} d \lambda$, where $Z$ is the normalization constant and $N^{-2 n}$ the fundamental solution. Let $g:[0, \infty) \rightarrow[0, \infty)$ be a differentiable increasing function such that $g^{\prime}(N)$ is increasing, $g(N) \leq\left(c \frac{g^{\prime}(N)}{N^{2}}\right)^{\frac{1}{\beta}}$, and $g^{\prime \prime}(N)<d g^{\prime}(N)^{2}$ on $\{N \geq 1\}$, for some constants $c$ and $d$. Then

$$
\mu\left(|f|^{q}\left|\log \left(\frac{|f|^{q}}{\mu|f|^{q}}\right)\right|^{\beta}\right) \leq C \mu|f|^{q}+D \mu|\nabla f|^{q},
$$

for $C$ and $D$ positive constants and for $q \geq 2$.

The proof follows closely that of Theorem 11 in [8], with some modification.

Proof Choose $\phi(x)=(1+x)^{\beta}$, which satisfies the conditions of Theorem 7. On $\{N \geq 1\}, g^{\prime \prime}(N)<d g^{\prime}(N)^{2} \leq g^{\prime}(N)^{3} N^{3}$, so the condition of Theorem 3 is satisfied. Thus, on $\{N \geq 1\}$, we have the U-bound (1.3):

$$
\mu\left(\frac{g^{\prime}(N)}{N^{2}}|f|^{q}\right) \leq C \mu|\nabla f|^{q}+D \mu|f|^{q} .
$$

By the condition $g(N) \leq\left(c \frac{g^{\prime}(N)}{N^{2}}\right)^{\frac{1}{\beta}}$, we obtain $\phi(g(N))=(1+g(N))^{\beta} \leq \frac{g^{\prime}(N)}{N^{2}}$ on $\{N \geq 1\}$. Hence, since $g(N)$ is increasing and using the U-bound,

$$
\begin{aligned}
\mu\left(\phi(g(N))|f|^{q}\right) & \leq \int_{\{N \geq 1\}}\left(\frac{g^{\prime}(N)}{N^{2}}|f|^{q}\right) d \mu+\int_{\{N<1\}} \phi(g(N))|f|^{q} d \mu \\
& \leq \int_{\{N \geq 1\}}\left(\frac{g^{\prime}(N)}{N^{2}}|f|^{q}\right) d \mu+\int_{\{N<1\}}(1+g(1))^{\beta}|f|^{q} d \mu \\
& \leq C \mu|\nabla f|^{q}+D \mu|f|^{q} .
\end{aligned}
$$

In order to use Theorem 8 , it remains to prove:

$$
\mu\left(|f|^{q}|\nabla g(N)|^{q}\right) \leq C \mu|f|^{q}+D \mu|\nabla f|^{q} .
$$

On $\{N<1\}$, since $g^{\prime}(N)$ is increasing and using (2.4), $\int_{\{N<1\}}\left(|f|^{q}|\nabla g(N)|^{q}\right) d \mu=$ $\int_{\{N<1\}}\left(|f|^{q}\left|g^{\prime}(N) \nabla N\right|^{q}\right) d \mu \leq \frac{\theta^{q}(2 n+1)^{q}}{2^{\frac{3 q}{2}} n^{q}} \int_{\{N<1\}}|f|^{q}\left|g^{\prime}(1)\right|^{q} d \mu$. 
We now need to consider $\{N \geq 1\}$ :

$$
\begin{aligned}
\int|f|^{q}(\nabla g(N) \cdot V-\nabla \cdot V) d \mu & =\int \nabla|f|^{q} \cdot V d \mu \leq \frac{\epsilon}{p} \int|f|^{q}|V|^{p} d \mu \\
& +\frac{1}{\epsilon^{\frac{q}{p}}} q^{q-1} \int|\nabla f|^{q} d \mu,
\end{aligned}
$$

where the last inequality uses $a b \leq \epsilon \frac{a^{p}}{p}+\frac{b^{q}}{\epsilon^{\frac{q}{p}} q}$, where $a=|f|^{q-1}|V|$, and $b=$ $q|\nabla f|$. Let $V=\nabla N \frac{|x|^{q-2}}{N^{q-2}} g^{\prime}(N)^{q-1}$. Since $\nabla g(N)=g^{\prime}(N) \nabla N$, then $\nabla g(N) \cdot V=$ $|\nabla g(N)|^{q}$, which is the term on the left hand side of (4.2). Using the inequality (2.4) on the first term on the right hand side of (4.3) we get

$$
\begin{aligned}
\frac{\epsilon}{p} \int|f|^{q}|V|^{p} d \mu & =\frac{\epsilon}{p} \int|\nabla N|^{p}|f|^{q} \frac{|x|^{(q-2) p} g^{\prime}(N)^{q}}{N^{(q-2) p}} d \mu \\
& \leq \frac{\epsilon}{p} \frac{\theta^{p}(2 n+1)^{p}}{2^{\frac{3 p}{2}} n^{p}} \int|f|^{q} \frac{|x|^{q} g^{\prime}(N)^{q}}{N^{q}} d \mu
\end{aligned}
$$

which can subtracted from the left hand side of (4.3) since by choosing $\epsilon$ small enough and noting that using (2.3),

$$
\nabla g(N) \cdot V=|\nabla N|^{2} \frac{|x|^{q-2}}{N^{q-2}} g^{\prime}(N)^{q} \geq \frac{\theta^{2}}{2^{5+\frac{2}{n}}} \frac{|x|^{q}}{N^{q}} g^{\prime}(N)^{q}
$$

It remains to compute $\nabla \cdot V$. Using the fact that $N^{-2 n}$ is the fundamental solution, $\triangle N=\frac{(Q-1)|\nabla N|^{2}}{N}, \quad Q=2 n+2$ the homogeneous dimension and $|\nabla N|^{2} \leq \frac{\theta^{2}(2 n+1)^{2}|x|^{2}}{2^{3} n^{2} N^{2}}(2.4)$,

$$
\begin{aligned}
\nabla \cdot V= & \Delta N \frac{|x|^{q-2}}{N^{q-2}} g^{\prime}(N)^{q-1}+(q-2) \frac{|x|^{q-4} g^{\prime}(N)^{q-1} x \cdot \nabla N}{N^{q-2}} \\
& -(q-2) \frac{|\nabla N|^{2}|x|^{q-2} g^{\prime}(N)^{q-1}}{N^{q-1}}+(q-1) g^{\prime}(N)^{q-2} g^{\prime \prime}(N) \frac{|x|^{q-2}|\nabla N|^{2}}{N^{q-2}} . \\
|\nabla \cdot V| \leq & \frac{(Q-1) \theta^{2}(2 n+1)^{2}}{2^{3} n^{2}} \frac{|x|^{q}}{N^{q+1}} g^{\prime}(N)^{q-1}+(q-2) \frac{\theta(2 n+1)|x|^{q-2} g^{\prime}(N)^{q-1}}{2^{\frac{3}{2}} n N^{q-1}} \\
& +(q-2) \frac{\theta^{2}(2 n+1)^{2}}{2^{3} n^{2}} \frac{|x|^{q} g^{\prime}(N)^{q-1}}{N^{q+1}}+(q-1) \frac{\theta^{2}(2 n+1)^{2}}{2^{3} n^{2}} g^{\prime}(N)^{q-2} g^{\prime \prime}(N) \frac{|x|^{q}}{N^{q}} .
\end{aligned}
$$

All terms can be absorbed by the first term in (4.3). Using (4.1) and (4.2), the condition of Theorem 8 is satisfied, and we obtain $\beta$-logarithmic Sobolev inequality:

$$
\mu\left(|f|^{q}\left|\log \left(\frac{|f|^{q}}{\mu|f|^{q}}\right)\right|^{\beta}\right) \leq C \mu|f|^{q}+D \mu|\nabla f|^{q}
$$


for $C$ and $D$ positive constants independent of $f$.

As a corollary, we obtain the following result:

Corollary 10 Let $\mathbb{R}^{2 n+1}$ be an anisotropic Heisenberg group with $n>5$ and $N$ the homogeneous norm corresponding to the fundamental solution of the sub-Laplacian equation. Let the probability measure be $d \mu=\frac{e^{-\beta N^{p}}}{Z} d \lambda$, where $Z$ is the normalization constant. Then, for $p \geq 4$ and $0<\beta \leq \frac{p-3}{p}$,

$$
\mu\left(|f|^{q}\left|\log \left(\frac{|f|^{q}}{\mu|f|^{q}}\right)\right|^{\beta}\right) \leq C \mu|f|^{q}+D \mu|\nabla f|^{q},
$$

for $C$ and $D$ positive constants and for $q \geq 2$.

Data Availability Data sharing is not applicable to this article as no datasets were generated or analysed during the current study.

\section{Declarations}

Conflict of interest The authors declare that they have no conflict of interest.

Open Access This article is licensed under a Creative Commons Attribution 4.0 International License, which permits use, sharing, adaptation, distribution and reproduction in any medium or format, as long as you give appropriate credit to the original author(s) and the source, provide a link to the Creative Commons licence, and indicate if changes were made. The images or other third party material in this article are included in the article's Creative Commons licence, unless indicated otherwise in a credit line to the material. If material is not included in the article's Creative Commons licence and your intended use is not permitted by statutory regulation or exceeds the permitted use, you will need to obtain permission directly from the copyright holder. To view a copy of this licence, visit http://creativecommons.org/licenses/by/4.0/.

\section{Appendix}

\section{Derivation of the formula for homogeneous norm $N$}

To compute explicitly the fundamental solution, we now use R. Beals, Gaveau, and Greiner's Theorem 2 of [3]. The fundamental solution for the sub-laplacian $\Delta=$ $\sum_{j=1}^{2 n} X_{j}^{2}$ on $\mathbb{G}$ with singularity at 0 is:

$$
u(x, t)=\frac{\Gamma\left(\frac{Q}{2}-1\right)}{2(2 \pi)^{\frac{Q}{2}}} \int_{\mathbb{R}} \frac{V(\tau)}{f(x, t, \tau)^{\frac{Q}{2}-1}} d \tau,
$$

for $(x, t) \in \mathbb{G}, \quad x \neq 0$. Where we have $Q=2 n+2$ the homogeneous dimension, $V: \mathbb{R} \rightarrow \mathbb{R}$ such that

$$
V(\tau)=\prod_{j=1}^{2 n} w_{j}(\tau)^{\frac{1}{2}} \operatorname{csch}\left(w_{j}(\tau)\right)^{\frac{1}{2}}
$$


and $f: \mathbb{G} \times \mathbb{R} \rightarrow \mathbb{C}$ such that

$$
f(x, t, \tau)=\frac{1}{2} \sum_{j=1}^{2 n} w_{j}(\tau) \operatorname{coth}\left(w_{j}(\tau)\right)\left|x \cdot e_{j}(\tau)\right|^{2}-\sqrt{-1} t \tau .
$$

Here, $w_{j}(\tau)$ are the eigenvalues and $e_{j}(\tau)$ are the corresponding normalised eigenvectors of the $2 n \times 2 n$ matrix $\Omega(\tau)=-\sqrt{-1} \tau M$, where

$$
M_{k l}= \begin{cases}-\frac{\theta}{2} & k=1, \quad l=n+1 \\ \frac{\theta}{2} & k=n+1, \quad l=1 \\ -\theta & k=2,3, \ldots, n, \quad l=k+n \\ \theta & k=n+2, n+3, \ldots, 2 n, \quad l=k-n \\ 0 & \text { otherwise }\end{cases}
$$

The eigenvalues are: $w_{1}(\tau)=-\frac{\theta \tau}{2}, w_{n+1}(\tau)=\frac{\theta \tau}{2}, w_{j}(\tau)=-\theta \tau$, for $j=$ $2,3, \ldots, n$, and $w_{j}(\tau)=\theta \tau$, for $j=n+2, n+3, \ldots, 2 n$. The corresponding normalised eigenvectors are:

$$
\left(e_{j}(\tau)\right)_{i}= \begin{cases}-\frac{\sqrt{-1}}{\sqrt{2}} & i=j \\ \frac{1}{\sqrt{2}} & i=j+n \\ 0 & \text { otherwise }\end{cases}
$$

for $j=1,2, \ldots, n$, and

$$
\left(e_{j}(\tau)\right)_{i}= \begin{cases}\frac{\sqrt{-1}}{\sqrt{2}} & i=j-n \\ \frac{1}{\sqrt{2}} & i=j \\ 0 & \text { otherwise }\end{cases}
$$

for $j=n+1, n+2, \ldots, 2 n$. Thus,

$$
\begin{aligned}
V(\tau)= & \prod_{j=1}^{2 n} w_{j}(\tau)^{\frac{1}{2}} \operatorname{csch}\left(w_{j}(\tau)\right)^{\frac{1}{2}}=\left(\frac{(\theta \tau)^{2}}{4} \operatorname{csch}\left(\frac{\theta \tau}{2}\right)^{2}(\theta \tau)^{2 n-2} \operatorname{csch}(\theta \tau)^{2 n-2}\right)^{\frac{1}{2}} \\
= & \frac{\theta^{n} \tau^{n}}{2} \operatorname{csch}\left(\frac{\theta \tau}{2}\right) \operatorname{csch}(\theta \tau)^{n-1}, \\
f(x, t, \tau)= & \frac{1}{2} \sum_{j=1}^{2 n} w_{j}(\tau) \operatorname{coth}\left(w_{j}(\tau)\right)\left|x \cdot e_{j}(\tau)\right|^{2}-\sqrt{-1} t \tau \\
= & \frac{1}{2} w_{1}(\tau) \operatorname{coth}\left(w_{1}(\tau)\right)\left|x \cdot e_{1}(\tau)\right|^{2}+\frac{1}{2} w_{n+1}(\tau) \operatorname{coth}\left(w_{n+1}(\tau)\right)\left|x \cdot e_{n+1}(\tau)\right|^{2} \\
& +\frac{1}{2} \sum_{j=2, j \neq n+1}^{2 n} w_{j}(\tau) \operatorname{coth}\left(w_{j}(\tau)\right)\left|x \cdot e_{j}(\tau)\right|^{2}-\sqrt{-1} t \tau=-\frac{\theta \tau}{4} \operatorname{coth}\left(-\frac{\theta \tau}{2}\right)\left|-\frac{\sqrt{-1}}{\sqrt{2}} x_{1}+\frac{x_{n+1}}{\sqrt{2}}\right|^{2} \\
& +\frac{\theta \tau}{4} \operatorname{coth}\left(\frac{\theta \tau}{2}\right)\left|\frac{\sqrt{-1}}{\sqrt{2}} x_{1}+\frac{x_{n+1}}{\sqrt{2}}\right|^{2}+\frac{1}{2} \sum_{j=2}^{n}-\theta \tau \operatorname{coth}(-\theta \tau)\left|-\frac{\sqrt{-1}}{\sqrt{2}} x_{j}+\frac{x_{j+n}}{\sqrt{2}}\right|^{2}
\end{aligned}
$$




$$
\begin{aligned}
& +\frac{1}{2} \sum_{j=n+2}^{2 n} \theta \tau \operatorname{coth}(\theta \tau)\left|\frac{\sqrt{-1}}{\sqrt{2}} x_{j}+\frac{x_{j+n}}{\sqrt{2}}\right|^{2}-\sqrt{-1} t \tau \\
= & \frac{\tau}{2} \operatorname{coth}\left(\frac{\theta \tau}{2}\right)\left(\frac{\theta x_{1}^{2}}{2}+\frac{\theta x_{n+1}^{2}}{2}\right)+\frac{\tau}{2} \operatorname{coth}(\theta \tau)\left(\sum_{j=2, j \neq n+1}^{2 n} \theta x_{j}^{2}\right)-\sqrt{-1} t \tau .
\end{aligned}
$$

Letting $A=\frac{\theta x_{1}^{2}}{2}+\frac{\theta x_{n+1}^{2}}{2}+\frac{\theta}{2} \sum_{j=2, j \neq n+1}^{2 n} x_{j}^{2}$ and $B=\frac{\theta x_{1}^{2}}{4}+\frac{\theta x_{n+1}^{2}}{4}+\frac{\theta}{2} \sum_{j=2, j \neq n+1}^{2 n} x_{j}^{2}$, we obtain:

$$
f(x, t, \tau)=\tau \operatorname{coth}\left(\frac{\theta \tau}{2}\right)(A-B)+\tau \operatorname{coth}(\theta \tau)(2 B-A)-\sqrt{-1} t \tau .
$$

Replacing (5.2) and (5.3) in (5.1), we get:

$$
\begin{aligned}
u(x, t) & =\frac{\Gamma(n)}{2(2 \pi)^{n+1}} \int_{\mathbb{R}} \frac{V(\tau)}{f(x, t, \tau)^{n}} d \tau \\
& =\frac{\Gamma(n)}{2(2 \pi)^{n+1}} \int_{\mathbb{R}} \frac{\frac{\theta^{n} \tau^{n}}{2} \operatorname{csch}\left(\frac{\theta \tau}{2}\right) \operatorname{csch}(\theta \tau)^{n-1}}{\left(\tau \operatorname{coth}\left(\frac{\theta \tau}{2}\right)(A-B)+\tau \operatorname{coth}(\theta \tau)(2 B-A)-\sqrt{-1} t \tau\right)^{n}} d \tau \\
& =\frac{\Gamma(n)}{(2 \pi)^{n+1}} \int_{0}^{\infty} \operatorname{Re}\left\{\frac{\frac{\theta^{n} \tau^{n}}{2} \operatorname{csch}\left(\frac{\theta \tau}{2}\right) \operatorname{csch}(\theta \tau)^{n-1}}{\left(\tau \operatorname{coth}\left(\frac{\theta \tau}{2}\right)(A-B)+\tau \operatorname{coth}(\theta \tau)(2 B-A)-\sqrt{-1} t \tau\right)^{n}}\right\} d \tau \\
& =\frac{\theta^{n} \Gamma(n)}{2(2 \pi)^{n+1}} \int_{0}^{\infty} \operatorname{Re}\left\{\frac{\operatorname{csch}\left(\frac{\theta \tau}{2}\right) \operatorname{csch}(\theta \tau)^{n-1} \operatorname{coth}\left(\frac{\theta \tau}{2}\right)^{n}}{\operatorname{coth}\left(\frac{\theta \tau}{2}\right)^{n}\left(\operatorname{coth}\left(\frac{\theta \tau}{2}\right)(A-B)+\operatorname{coth}(\theta \tau)(2 B-A)-\sqrt{-1} t\right)^{n}}\right\} d \tau
\end{aligned}
$$

using the trigonometric identity $\operatorname{coth}(\theta \tau)=\frac{1}{2 \operatorname{coth}\left(\frac{\theta \tau}{2}\right)}+\frac{\operatorname{coth}\left(\frac{\theta \tau}{2}\right)}{2}$,

$$
=\frac{\theta^{n} \Gamma(n)}{2(2 \pi)^{n+1}} \int_{0}^{\infty} \operatorname{Re}\left\{\frac{\operatorname{csch}\left(\frac{\theta \tau}{2}\right) \operatorname{csch}(\theta \tau)^{n-1} \operatorname{coth}\left(\frac{\theta \tau}{2}\right)^{n}}{\left(\frac{A}{2} \operatorname{coth}\left(\frac{\theta \tau}{2}\right)^{2}+B-\frac{A}{2}-\sqrt{-1} t \operatorname{coth}\left(\frac{\theta \tau}{2}\right)\right)^{n}}\right\} d \tau
$$

using the trigonometric identities $\operatorname{csch}(\theta \tau)=\frac{\left(\operatorname{csch}\left(\frac{\theta \tau}{2}\right)\right)^{2}}{2 \operatorname{coth}\left(\frac{\theta \tau}{2}\right)}$ and $\operatorname{coth}\left(\frac{\theta \tau}{2}\right)^{2}=1$ $+\operatorname{csch}\left(\frac{\theta \tau}{2}\right)^{2}$

$$
=\frac{\theta^{n} \Gamma(n)}{(2 \pi)^{n+1}} \int_{0}^{\infty} \operatorname{Re}\left\{\frac{\operatorname{csch}\left(\frac{\theta \tau}{2}\right) \operatorname{csch}\left(\frac{\theta \tau}{2}\right)^{2 n-2} \operatorname{coth}\left(\frac{\theta \tau}{2}\right)}{\left(A \operatorname{csch}\left(\frac{\theta \tau}{2}\right)^{2}+2 B-2 \sqrt{-1} t \sqrt{1+\operatorname{csch}\left(\frac{\theta \tau}{2}\right)^{2}}\right)^{n}}\right\} d \tau
$$

letting $u=\operatorname{csch}\left(\frac{\theta \tau}{2}\right)$, we have $d u=-\frac{\theta}{2} \operatorname{coth}\left(\frac{\theta \tau}{2}\right) \operatorname{csch}\left(\frac{\theta \tau}{2}\right) d \tau$

$$
=\frac{\theta^{n-1} \Gamma(n)}{\pi(2 \pi)^{n}} \operatorname{Re}\left\{\int_{0}^{\infty} \frac{u^{2 n-2}}{\left(A u^{2}+2 B-2 \sqrt{-1} t \sqrt{1+u^{2}}\right)^{n}} d u\right\}
$$




$$
\begin{gathered}
=\frac{\theta^{n-1} \Gamma(n)}{\pi(2 \pi)^{n}} \operatorname{Re}\left\{\frac{(-1)^{n-2}}{(n-1) !} \frac{\partial^{n-2}}{\partial A^{n-2}} \int_{0}^{\infty} \frac{u^{2}}{\left(A u^{2}+2 B-2 \sqrt{-1} t \sqrt{1+u^{2}}\right)^{2}} d u\right\} \\
=\frac{(-1)^{n-2} \theta^{n-1}}{\pi(2 \pi)^{n}} \frac{d^{n-2}}{d A^{n-2}} \operatorname{Re}\left\{\int_{0}^{\infty} \frac{u^{2}}{\left(A u^{2}+2 B-2 \sqrt{-1} t \sqrt{1+u^{2}}\right)^{2}} d u\right\} .
\end{gathered}
$$

The next step is to compute

$$
\begin{aligned}
I(A, B, t) & =\operatorname{Re}\left\{\int_{0}^{\infty} \frac{u^{2}}{\left(A u^{2}+2 B-2 \sqrt{-1} t \sqrt{1+u^{2}}\right)^{2}} d u\right\} \\
& =\int_{0}^{\infty} \frac{\left(A u^{2}+2 B\right)^{2} u^{2}-4 t^{2}\left(1+u^{2}\right) u^{2}}{\left(\left(A u^{2}+2 B\right)^{2}+4 t^{2}\left(1+u^{2}\right)\right)^{2}} d u .
\end{aligned}
$$

Write $I(A, B, t)=I_{1}(A, B, t)-I_{2}(A, B, t)$, where

$$
I_{1}(A, B, t)=\int_{0}^{\infty} \frac{u^{2}}{\left(A u^{2}+2 B\right)^{2}+4 t^{2}\left(1+u^{2}\right)} d u
$$

and

$$
I_{2}(A, B, t)=\int_{0}^{\infty} \frac{8 t^{2}\left(1+u^{2}\right) u^{2}}{\left(\left(A u^{2}+2 B\right)^{2}+4 t^{2}\left(1+u^{2}\right)\right)^{2}} d u
$$

Remark that

$$
I_{2}(A, B, t)=-t \partial_{t} I_{1}(A, B, t)
$$

so

$$
I(A, B, t)=I_{1}(A, B, t)+t \partial_{t} I_{1}(A, B, t) .
$$

We now compute $I_{1}(A, B, t)=\frac{1}{2} \int_{-\infty}^{\infty} \frac{x^{2}}{\left(A x^{2}+2 B\right)^{2}+4 t^{2}\left(1+x^{2}\right)} d x$ using complex theory. Write denominator in the integrand as a polynomial

$$
p(z)=\left(A z^{2}+2 B\right)^{2}+4 t^{2}\left(1+z^{2}\right)=A^{2} z^{4}+\left(4 A B+4 t^{2}\right) z^{2}+4 B^{2}+4 t^{2} .
$$

$p(z)$ has four complex roots. If $z$ is a root, then $-z$ is a root. Since $p(z)$ has real coefficients, then $\bar{z}$ is a root, and $-\bar{z}$ is the fourth root. Let $\alpha$ be a root in the upper half 
plane, so $-\bar{\alpha}$ is also in the upper half plane. We thus have

$$
p(z)=A^{2}(z-\alpha)(z+\alpha)(z-\bar{\alpha})(z+\bar{\alpha})=A^{2}\left(z^{4}-\left(\alpha^{2}+\bar{\alpha}^{2}\right) z^{2}+|\alpha|^{4}\right) .
$$

By identification with (5.6), we get that $\alpha^{2}+\bar{\alpha}^{2}=-\frac{4 A B+4 t^{2}}{A^{2}}$ and $|\alpha|^{4}=\frac{4 B^{2}+4 t^{2}}{A^{2}}$. From this, we can calculate $\operatorname{Im}(\alpha)=\frac{\alpha-\bar{\alpha}}{2 i}$. In fact,

$$
\operatorname{Im}(\alpha)^{2}=-\frac{\alpha^{2}+\bar{\alpha}^{2}-2|\alpha|^{2}}{4}=\frac{A B+t^{2}+A \sqrt{B^{2}+t^{2}}}{A^{2}} .
$$

Now, we go back to computing the integral: Let $f(z)=\frac{z^{2}}{\left(A z^{2}+2 B\right)^{2}+4 t^{2}\left(1+z^{2}\right)}$, by the residue theorem:

$$
\begin{aligned}
I_{1}(A, B, t) & =\pi i(\operatorname{Res}(f, \alpha)+\operatorname{Res}(f,-\bar{\alpha})) \\
\operatorname{Res}(f, \alpha) & =\lim _{z \rightarrow \alpha}(z-\alpha) f(z)=\lim _{z \rightarrow \alpha}(z-\alpha) \frac{z^{2}}{A^{2}(z-\alpha)(z+\alpha)(z-\bar{\alpha})(z+\bar{\alpha})} \\
& =\frac{\alpha}{2 A^{2}\left(\alpha^{2}-\bar{\alpha}^{2}\right)}
\end{aligned}
$$

and

$$
\begin{aligned}
\operatorname{Res}(f,-\bar{\alpha}) & =\lim _{z \rightarrow-\bar{\alpha}}(z+\bar{\alpha}) f(z)=\lim _{z \rightarrow-\bar{\alpha}}(z+\bar{\alpha}) \frac{z^{2}}{A^{2}(z-\alpha)(z+\alpha)(z-\bar{\alpha})(z+\bar{\alpha})} \\
& =\frac{\bar{\alpha}}{2 A^{2}\left(\alpha^{2}-\bar{\alpha}^{2}\right)} .
\end{aligned}
$$

Replacing in (5.8), we get:

$$
\begin{aligned}
I_{1}(A, B, t) & =\pi i(\operatorname{Res}(f, \alpha)+\operatorname{Res}(f,-\bar{\alpha})) \\
& =\pi i\left(\frac{\alpha}{2 A^{2}\left(\alpha^{2}-\bar{\alpha}^{2}\right)}+\frac{\bar{\alpha}}{2 A^{2}\left(\alpha^{2}-\bar{\alpha}^{2}\right)}\right) \\
& =\frac{2 \pi i}{4 A^{2}(\alpha-\bar{\alpha})}=\frac{\pi}{4 A^{2} \operatorname{Im}(\alpha)}
\end{aligned}
$$

using (5.7)

$$
=\frac{\pi}{4 A\left(A B+t^{2}+A \sqrt{B^{2}+t^{2}}\right)^{\frac{1}{2}}} .
$$

We can now compute $I(A, B, t)$ using (5.5):

$$
\begin{aligned}
I(A, B, t) & =I_{1}(A, B, t)+t \partial_{t} I_{1}(A, B, t) \\
& =\frac{\pi}{4 A\left(A B+t^{2}+A \sqrt{B^{2}+t^{2}}\right)^{\frac{1}{2}}}+t \partial_{t} \frac{\pi}{4 A\left(A B+t^{2}+A \sqrt{B^{2}+t^{2}}\right)^{\frac{1}{2}}}
\end{aligned}
$$




$$
\begin{aligned}
& =\frac{\pi}{4 A\left(A B+t^{2}+A \sqrt{B^{2}+t^{2}}\right)^{\frac{1}{2}}}-\frac{\pi\left(2 t^{2} \sqrt{B^{2}+t^{2}}+A t^{2}\right)}{8 A \sqrt{B^{2}+t^{2}}\left(A B+t^{2}+A \sqrt{B^{2}+t^{2}}\right)^{\frac{3}{2}}} \\
& =\pi \frac{2 \sqrt{B^{2}+t^{2}}\left(A B+t^{2}+A \sqrt{B^{2}+t^{2}}\right)-2 t^{2} \sqrt{B^{2}+t^{2}}-A t^{2}}{8 A \sqrt{B^{2}+t^{2}}\left(A B+t^{2}+A \sqrt{B^{2}+t^{2}}\right)^{\frac{3}{2}}} \\
& =\pi \frac{2 B \sqrt{B^{2}+t^{2}}+2 B^{2}+t^{2}}{8 \sqrt{B^{2}+t^{2}}\left(A B+t^{2}+A \sqrt{B^{2}+t^{2}}\right)^{\frac{3}{2}}} \\
& =\frac{\pi\left(B+\sqrt{B^{2}+t^{2}}\right)^{2}}{8 \sqrt{B^{2}+t^{2}}\left(A B+t^{2}+A \sqrt{B^{2}+t^{2}}\right)^{\frac{3}{2}}} .
\end{aligned}
$$

Using (5.4),

$$
\begin{aligned}
u(x, t) & =\frac{(-1)^{n-2} \theta^{n-1}}{\pi(2 \pi)^{n}} \frac{d^{n-2}}{d A^{n-2}} I(A, B, t) \\
& =\frac{(-1)^{n-2} \theta^{n-1}}{(2 \pi)^{n}} \frac{d^{n-2}}{d A^{n-2}}\left(\frac{\left(B+\sqrt{B^{2}+t^{2}}\right)^{2}}{8 \sqrt{B^{2}+t^{2}}\left(A B+t^{2}+A \sqrt{B^{2}+t^{2}}\right)^{\frac{3}{2}}}\right) \\
& =\frac{(-1)^{n-2}(-1)^{n-2} \theta^{n-1} \prod_{k=3}^{n}(2 k-3)}{(2 \pi)^{n} 2^{n-2}}\left(\frac{\left(B+\sqrt{B^{2}+t^{2}}\right)^{2+n-2}}{8 \sqrt{B^{2}+t^{2}}\left(A B+t^{2}+A \sqrt{B^{2}+t^{2}}\right)^{\frac{3}{2}+n-2}}\right) \\
& =\frac{\theta^{n-1} \prod_{k=3}^{n}(2 k-3)}{(2 \pi)^{n} 2^{n+1}}\left(\frac{\left.\sqrt{B^{2}+t^{2}}\right)^{n}}{\sqrt{B^{2}+t^{2}}\left(A B+t^{2}+A \sqrt{B^{2}+t^{2}}\right)^{n-\frac{1}{2}}}\right) .
\end{aligned}
$$

The fundamental solution is given up to a constant multiple:

$$
N(x, t)=u(x, t)^{\frac{1}{2-Q}}=u(x, t)^{-\frac{1}{2 n}}
$$

using the last equation,

$$
N(x, t)=\frac{\left(B^{2}+t^{2}\right)^{\frac{1}{4 n}}\left(A B+t^{2}+A \sqrt{B^{2}+t^{2}}\right)^{\frac{1}{2}-\frac{1}{4 n}}}{\left(B+\sqrt{B^{2}+t^{2}}\right)^{\frac{1}{2}}}
$$

where $A=\frac{\theta x_{1}^{2}}{2}+\frac{\theta x_{n+1}^{2}}{2}+\frac{\theta}{2} \sum_{j=2, j \neq n+1}^{2 n} x_{j}^{2}$ and $B=\frac{\theta x_{1}^{2}}{4}+\frac{\theta x_{n+1}^{2}}{4}+\frac{\theta}{2} \sum_{j=2, j \neq n+1}^{2 n} x_{j}^{2}$. Note that for $n=2$ and $\theta=2$, and by using $\left(A B+t^{2}+A \sqrt{B^{2}+t^{2}}\right)=$ $\left(B+\sqrt{B^{2}+t^{2}}\right)\left(A-B+\sqrt{B^{2}+t^{2}}\right)$, we get back the fundamental solution as calculated in [2]. 


\section{Proof of Lemma 2: Bounds for $|\nabla \boldsymbol{N}|$ and $x \cdot \nabla \boldsymbol{N}$}

Recall that by $|x|=\left(\sum_{j=1}^{2 n} x_{j}^{2}\right)^{\frac{1}{2}}$ we denote the Euclidean norm. In the setting of the anisotropic Heisenberg group $\mathbb{R}^{2 n+1}$, we have the fundamental solution

$$
N=\left(\frac{\left(B^{2}+t^{2}\right)^{\frac{1}{2 n}}\left(A B+t^{2}+A \sqrt{B^{2}+t^{2}}\right)^{1-\frac{1}{2 n}}}{B+\sqrt{B^{2}+t^{2}}}\right)^{\frac{1}{2}},
$$

where $A=\frac{\theta x_{1}^{2}}{2}+\frac{\theta x_{n+1}^{2}}{2}+\frac{\theta}{2} \sum_{j=2, j \neq n+1}^{2 n} x_{j}^{2}$ and $B=\frac{\theta x_{1}^{2}}{4}+\frac{\theta x_{n+1}^{2}}{4}+\frac{\theta}{2} \sum_{j=2, j \neq n+1}^{2 n} x_{j}^{2}$. In this subsection, we are going to show the following relations:

$$
\begin{aligned}
& x \cdot \nabla N \geq-\frac{\theta|x|^{2}}{4 n N}, \\
& |\nabla N|^{2} \geq \frac{\theta^{2}|x|^{2}}{2^{5+\frac{2}{n}} N^{2}},
\end{aligned}
$$

and

$$
|\nabla N|^{2} \leq \frac{(2 n+1)^{2} \theta^{2}|x|^{2}}{2^{3} n^{2} N^{2}}
$$

Proof We first calculate $\partial_{x_{j}} N$ and $\partial_{t} N$.

For $j=1$ and $j=n+1$ :

$$
\begin{aligned}
\partial_{x_{j}} N= & \frac{\theta x_{j}}{4 n N}\left(\frac{B\left(B^{2}+t^{2}\right)^{\frac{1}{2 n}}\left(A B+t^{2}+A \sqrt{B^{2}+t^{2}}\right)}{\left(B+\sqrt{B^{2}+t^{2}}\right)\left(B^{2}+t^{2}\right)\left(A B+t^{2}+A \sqrt{B^{2}+t^{2}}\right)^{\frac{1}{2 n}}}\right) \\
& +\frac{\theta x_{j}(2 n-1)}{4 n N}\left(\frac{\left(B^{2}+t^{2}\right)^{\frac{1}{2 n}}\left(\frac{A}{2} \sqrt{B^{2}+t^{2}}+B \sqrt{B^{2}+t^{2}}+B^{2}+t^{2}+\frac{A B}{2}\right)}{\left(B+\sqrt{B^{2}+t^{2}}\right)\left(A B+t^{2}+A \sqrt{B^{2}+t^{2}}\right)^{\frac{1}{2 n}}\left(B^{2}+t^{2}\right)^{\frac{1}{2}}}\right) \\
& -\frac{\theta x_{j}}{4 N}\left(\frac{\left(B^{2}+t^{2}\right)^{\frac{1}{2 n}}\left(A B+t^{2}+A \sqrt{B^{2}+t^{2}}\right)}{\left(B+\sqrt{B^{2}+t^{2}}\right)\left(B^{2}+t^{2}\right)^{\frac{1}{2}}\left(A B+t^{2}+A \sqrt{B^{2}+t^{2}}\right)^{\frac{1}{2 n}}}\right) \\
= & \frac{x_{j}\left(B^{2}+t^{2}\right)^{\frac{1}{2 n}}}{4 n N} \\
& \cdot\left(\frac{\frac{1}{2} B^{2} A+\left(B-\frac{A}{2}\right) t^{2}+\frac{1}{2} \sqrt{B^{2}+t^{2}} A B+(n-1)\left(B^{2}+t^{2}\right)\left(B+\sqrt{B^{2}+t^{2}}\right)+n B \sqrt{B^{2}+t^{2}}\left(B+\sqrt{B^{2}+t^{2}}\right)}{\left(B+\sqrt{B^{2}+t^{2}}\right)\left(B^{2}+t^{2}\right)\left(A B+t^{2}+A \sqrt{B^{2}+t^{2}}\right)^{\frac{1}{2 n}}}\right)
\end{aligned}
$$

Notice that

$$
x_{j} \partial_{x_{j}} N \geq 0 \quad \text { for } j=1, n+1
$$


To get a bound from above for $\left|\partial_{x_{j}} N\right|$, since $B \leq \sqrt{B^{2}+t^{2}}, B-\frac{A}{2} \leq \frac{A}{2}$, and $B^{2}+t^{2} \leq A B+t^{2}+A \sqrt{B^{2}+t^{2}}$,

$$
\left|\partial_{x_{j}} N\right| \leq \frac{\theta\left|x_{j}\right|}{4 n N}\left(\frac{\frac{1}{2} B^{2} A+\left(\frac{A}{2}\right) t^{2}+\frac{1}{2} \sqrt{B^{2}+t^{2}} A B+(2 n-1)\left(B^{2}+t^{2}\right)\left(B+\sqrt{B^{2}+t^{2}}\right)}{\left(B+\sqrt{B^{2}+t^{2}}\right)\left(B^{2}+t^{2}\right)}\right)
$$

since $A \leq 2 B$, so $\frac{1}{2} B^{2} A+\left(\frac{A}{2}\right) t^{2}+\frac{1}{2} \sqrt{B^{2}+t^{2}} A B \leq B \sqrt{B^{2}+t^{2}}\left(B+\sqrt{B^{2}+t^{2}}\right)$ $\leq\left(B^{2}+t^{2}\right)\left(B+\sqrt{B^{2}+t^{2}}\right)$,

$$
\left|\partial_{x_{j}} N\right| \leq \frac{\theta\left|x_{j}\right|}{2 N} \quad \text { for } j=1, n+1
$$

Now we calculate $\partial_{x_{j}} N$ for $j=2, \ldots, n, n+2, \ldots, 2 n$ :

$$
\begin{aligned}
\partial_{x_{j}} N= & \frac{\theta x_{j}}{4 n N}\left(\frac{2 B\left(B^{2}+t^{2}\right)^{\frac{1}{2 n}}\left(A B+t^{2}+A \sqrt{B^{2}+t^{2}}\right)}{\left(B+\sqrt{B^{2}+t^{2}}\right)\left(B^{2}+t^{2}\right)\left(A B+t^{2}+A \sqrt{B^{2}+t^{2}}\right)^{\frac{1}{2 n}}}\right) \\
& +\frac{\theta x_{j}(2 n-1)}{4 n N}\left(\frac{\left(B^{2}+t^{2}\right)^{\frac{1}{2 n}}\left(A \sqrt{B^{2}+t^{2}}+B \sqrt{B^{2}+t^{2}}+B^{2}+t^{2}+A B\right)}{\left(B+\sqrt{B^{2}+t^{2}}\right)\left(A B+t^{2}+A \sqrt{B^{2}+t^{2}}\right)^{\frac{1}{2 n}}\left(B^{2}+t^{2}\right)^{\frac{1}{2}}}\right) \\
& -\frac{\theta x_{j}}{4 N}\left(\frac{2\left(B^{2}+t^{2}\right)^{\frac{1}{2 n}}\left(A B+t^{2}+A \sqrt{B^{2}+t^{2}}\right)}{\left(B+\sqrt{B^{2}+t^{2}}\right)\left(B^{2}+t^{2}\right)^{\frac{1}{2}}\left(A B+t^{2}+A \sqrt{B^{2}+t^{2}}\right)^{\frac{1}{2 n}}}\right)=\frac{\theta x_{j}\left(B^{2}+t^{2}\right)^{\frac{1}{2 n}}}{4 n N} \\
& \left(\frac{A B \sqrt{B^{2}+t^{2}}+A B^{2}+(2 B-A) t^{2}+(2 n-1) B \sqrt{B^{2}+t^{2}}\left(B+\sqrt{B^{2}+t^{2}}\right)-t^{2} \sqrt{B^{2}+t^{2}}}{\left(B+\sqrt{B^{2}+t^{2}}\right)\left(B^{2}+t^{2}\right)\left(A B+t^{2}+A \sqrt{B^{2}+t^{2}}\right)^{\frac{1}{2 n}}}\right)
\end{aligned}
$$

Notice that

$$
x_{j} \partial_{x_{j}} N \geq \frac{\theta x_{j}^{2}\left(B^{2}+t^{2}\right)^{\frac{1}{2 n}}}{4 n N}\left(\frac{-t^{2}}{\left(B+\sqrt{B^{2}+t^{2}}\right) \sqrt{B^{2}+t^{2}}\left(A B+t^{2}+A \sqrt{B^{2}+t^{2}}\right)^{\frac{1}{2 n}}}\right)
$$

since $t^{2} \leq\left(B+\sqrt{B^{2}+t^{2}}\right) \sqrt{B^{2}+t^{2}}$ and $B^{2}+t^{2} \leq A B+t^{2}+A \sqrt{B^{2}+t^{2}}$

$$
x_{j} \partial_{x_{j}} N \geq-\frac{\theta x_{j}^{2}}{4 n N}
$$

We will now be able to get a lower bound for $x \cdot \nabla N$. In fact,

$$
x \cdot \nabla N=x_{1}\left(\partial_{x_{1}} N-\frac{\theta x_{n+1}}{2} \partial_{t} N\right)+x_{n+1}\left(\partial_{x_{n+1}} N+\frac{\theta x_{1}}{2} \partial_{t} N\right)
$$




$$
\begin{aligned}
& +\sum_{j=2}^{n} x_{j}\left(\partial_{x_{j}} N-\theta x_{j+n} \partial_{t} N\right)+\sum_{j=n+2}^{2 n} x_{j}\left(\partial_{x_{j}} N+\theta x_{j-n} \partial_{t} N\right) \\
& =x_{1} \partial_{x_{1}} N+x_{n+1} \partial_{x_{n+1}} N+\sum_{j=2, j \neq n+1}^{2 n} x_{j} \partial_{x_{j}} N
\end{aligned}
$$

using (5.14) and (5.17),

$$
\begin{aligned}
& \geq-\sum_{j=2, j \neq n+1}^{2 n} \frac{\theta x_{j}^{2}}{4 n N} \\
& \geq-\sum_{j=1}^{2 n} \frac{\theta x_{j}^{2}}{4 n N} .
\end{aligned}
$$

Using the above defined Euclidean norm $|x|=\left(\sum_{j=1}^{2 n} x_{j}^{2}\right)^{\frac{1}{2}}$,

$$
x \cdot \nabla N \geq-\frac{\theta|x|^{2}}{4 n N} .
$$

It remains to bound $|\nabla N|$.

$$
\begin{aligned}
|\nabla N|^{2}= & \left(\partial_{x_{1}} N-\frac{\theta x_{n+1}}{2} \partial_{t} N\right)^{2}+\left(\partial_{x_{n+1}} N+\frac{\theta x_{1}}{2} \partial_{t} N\right)^{2}+\sum_{j=2}^{n}\left(\partial_{x_{j}} N-\theta x_{j+n} \partial_{t} N\right)^{2} \\
& +\sum_{j=n+2}^{2 n}\left(\partial_{x_{j}} N+\theta x_{j-n} \partial_{t} N\right)^{2} \\
= & \sum_{j=1}^{2 n}\left(\partial_{x_{j}} N\right)^{2}+\frac{\theta^{2} x_{1}^{2}}{4}\left(\partial_{t} N\right)^{2}+\frac{\theta^{2} x_{n+1}^{2}}{4}\left(\partial_{t} N\right)^{2}+\sum_{j=2, j \neq n+1}^{2 n} \theta^{2} x_{j}^{2}\left(\partial_{t} N\right)^{2}
\end{aligned}
$$

To bound (5.18) from below, we use the fact that for each $j=2,3, \ldots, n, n+$ $2, \ldots, 2 n$,

$$
\left|\partial_{x_{j}} N\right|^{2}+\theta^{2} x_{j}^{2}\left|\partial_{t} N\right|^{2} \geq \frac{1}{2}\left(\left|\partial_{x_{j}} N\right|+\theta\left|x_{j}\right|\left|\partial_{t} N\right|\right)^{2},
$$

in addition to that we have for $j=1, n+1$,

$$
\left|\partial_{x_{j}} N\right|^{2}+\frac{\theta^{2} x_{j}^{2}}{4}\left|\partial_{t} N\right|^{2} \geq \frac{1}{2}\left(\left|\partial_{x_{j}} N\right|+\frac{\theta\left|x_{j}\right|}{2}\left|\partial_{t} N\right|\right)^{2}
$$

so,

$$
|\nabla N|^{2} \geq \frac{1}{2}\left(\left|\partial_{x_{1}} N\right|+\frac{\theta\left|x_{1}\right|}{2}\left|\partial_{t} N\right|\right)^{2}+\frac{1}{2}\left(\left|\partial_{x_{n+1}} N\right|+\frac{\theta\left|x_{n+1}\right|}{2}\left|\partial_{t} N\right|\right)^{2}
$$




$$
+\frac{1}{2} \sum_{j=2, j \neq n+1}^{2 n}\left(\left|\partial_{x_{j}} N\right|+\theta\left|x_{j} \| \partial_{t} N\right|\right)^{2}
$$

Now we proceed to calculate $\partial_{t} N$ :

$$
\begin{aligned}
\partial_{t} N= & \frac{1}{2 N}\left(\frac{t}{2 n} \frac{2\left(B^{2}+t^{2}\right)^{\frac{1}{2 n}}\left(A B+t^{2}+A \sqrt{B^{2}+t^{2}}\right)}{\left(B+\sqrt{B^{2}+t^{2}}\right)\left(B^{2}+t^{2}\right)\left(A B+t^{2}+A \sqrt{B^{2}+t^{2}}\right)^{\frac{1}{2 n}}}\right) \\
& +\frac{1}{2 N}\left(t\left(\frac{2 n-1}{2 n}\right) \frac{\left(B^{2}+t^{2}\right)^{\frac{1}{2 n}}\left(2 \sqrt{B^{2}+t^{2}}+A\right)}{\left(B+\sqrt{B^{2}+t^{2}}\right)\left(\sqrt{B^{2}+t^{2}}\right)\left(A B+t^{2}+A \sqrt{B^{2}+t^{2}}\right)^{\frac{1}{2 n}}}\right) \\
& -\frac{t}{4 N}\left(\frac{2\left(B^{2}+t^{2}\right)^{\frac{1}{2 n}}\left(A B+t^{2}+A \sqrt{B^{2}+t^{2}}\right)}{\left(B+\sqrt{B^{2}+t^{2}}\right)^{2}\left(\sqrt{B^{2}+t^{2}}\right)\left(A B+t^{2}+A \sqrt{B^{2}+t^{2}}\right)^{\frac{1}{2 n}}}\right) \\
= & \frac{t\left(B^{2}+t^{2}\right)^{\frac{1}{2 n}}}{4 n N}\left(\frac{2\left(A B+t^{2}+A \sqrt{B^{2}+t^{2}}\right)+(2 n-1) \sqrt{B^{2}+t^{2}}\left(2 \sqrt{B^{2}+t^{2}}+A\right)-2 n \frac{\sqrt{B^{2}+t^{2}}\left(A B+t^{2}+A \sqrt{B^{2}+t^{2}}\right)}{\left(B+\sqrt{B^{2}+t^{2}}\right)}}{\left(B+\sqrt{B^{2}+t^{2}}\right)\left(B^{2}+t^{2}\right)\left(A B+t^{2}+A \sqrt{B^{2}+t^{2}}\right)^{\frac{1}{2 n}}}\right) \\
= & \frac{t\left(B^{2}+t^{2}\right)^{\frac{1}{2 n}}}{4 n N}\left(\frac{2 B(A-B)+A \sqrt{B^{2}+t^{2}}+2 n \frac{2 B^{3}+2 t^{2} B+2 B^{2} \sqrt{B^{2}+t^{2}}+t^{2} \sqrt{B^{2}+t^{2}}}{\left(B+\sqrt{B^{2}+t^{2}}\right)}}{\left(B+\sqrt{B^{2}+t^{2}}\right)\left(B^{2}+t^{2}\right)\left(A B+t^{2}+A \sqrt{B^{2}+t^{2}}\right)^{\frac{1}{2 n}}}\right) .
\end{aligned}
$$

For $j=2, \ldots, n, n+2, \ldots, 2 n$, we use the reverse triangle inequality on (5.16) in addition to (5.20),

$$
\begin{aligned}
& \left|\partial_{x_{j}} N\right|+\theta\left|x_{j}\right|\left|\partial_{t} N\right| \geq \frac{\theta\left|x_{j}\right|\left(B^{2}+t^{2}\right)^{\frac{1}{2 n}}}{4 n N} \\
& \left(\frac{A B \sqrt{B^{2}+t^{2}}+A B^{2}+(2 B-A) t^{2}+(2 n-1) B \sqrt{B^{2}+t^{2}}\left(B+\sqrt{B^{2}+t^{2}}\right)-t^{2} \sqrt{B^{2}+t^{2}}}{\left(B+\sqrt{B^{2}+t^{2}}\right)\left(B^{2}+t^{2}\right)\left(A B+t^{2}+A \sqrt{B^{2}+t^{2}}\right)^{\frac{1}{2 n}}}\right) \\
& +\frac{\theta\left|x_{j}\right|\left(B^{2}+t^{2}\right)^{\frac{1}{2 n}}}{4 n N}\left(\frac{2 B(A-B)|t|+A|t| \sqrt{B^{2}+t^{2}}+2 n|t| \frac{2 B^{3}+2 t^{2} B+2 B^{2} \sqrt{B^{2}+t^{2}}+t^{2} \sqrt{B^{2}+t^{2}}}{\left(B+\sqrt{B^{2}+t^{2}}\right)}}{\left(B+\sqrt{B^{2}+t^{2}}\right)\left(B^{2}+t^{2}\right)\left(A B+t^{2}+A \sqrt{B^{2}+t^{2}}\right)^{\frac{1}{2 n}}}\right) \\
& =\frac{\theta\left|x_{j}\right|\left(B^{2}+t^{2}\right)^{\frac{1}{2 n}}}{4 n N} \\
& \left(\frac{A B \sqrt{B^{2}+t^{2}}+A|t| \sqrt{B^{2}+t^{2}}+A B^{2}+2 A B|t|-A t^{2}-B^{2} \sqrt{B^{2}+t^{2}}-t^{2} \sqrt{B^{2}+t^{2}}-2 B^{2}|t|-B^{3}+B t^{2}}{\left(B+\sqrt{B^{2}+t^{2}}\right)\left(B^{2}+t^{2}\right)\left(A B+t^{2}+A \sqrt{B^{2}+t^{2}}\right)^{\frac{1}{2 n}}}\right) \\
& +\frac{\theta\left|x_{j}\right|\left(B^{2}+t^{2}\right)^{\frac{1}{2 n}}}{4 n N}\left(\frac{n\left(2 B^{2} \sqrt{B^{2}+t^{2}}+2 B|t| \sqrt{B^{2}+t^{2}}+2 B^{2}|t|+2 B^{3}+2 B t^{2}+2|t|^{3}\right)}{\left(B+\sqrt{B^{2}+t^{2}}\right)\left(B^{2}+t^{2}\right)\left(A B+t^{2}+A \sqrt{B^{2}+t^{2}}\right)^{\frac{1}{2 n}}}\right)
\end{aligned}
$$

since $A|t| \sqrt{B^{2}+t^{2}} \geq A t^{2}, A B \sqrt{B^{2}+t^{2}} \geq B^{2} \sqrt{B^{2}+t^{2}}, A B^{2} \geq B^{3}$, and $2 A B|t| \geq 2 B^{2}|t|$,

$$
\geq \frac{\theta\left|x_{j}\right|\left(B^{2}+t^{2}\right)^{\frac{1}{2 n}}}{4 n N}\left(\frac{-t^{2} \sqrt{B^{2}+t^{2}}+B t^{2}+2 n\left(B^{2} \sqrt{B^{2}+t^{2}}+B|t| \sqrt{B^{2}+t^{2}}+B^{2} t+B^{3}+t^{2}(B+|t|)\right)}{\left(B+\sqrt{B^{2}+t^{2}}\right)\left(B^{2}+t^{2}\right)\left(A B+t^{2}+A \sqrt{B^{2}+t^{2}}\right)^{\frac{1}{2 n}}}\right)
$$


since $B+|t| \geq \sqrt{B^{2}+t^{2}}$,

$$
\geq \frac{\theta\left|x_{j}\right|(2 n-1)\left(B^{2}+t^{2}\right)^{\frac{1}{2 n}}}{4 n N}\left(\frac{B \sqrt{B^{2}+t^{2}}(B+|t|)+B^{2}(|t|+B)+t^{2} \sqrt{B^{2}+t^{2}}}{\left(B+\sqrt{B^{2}+t^{2}}\right)\left(B^{2}+t^{2}\right)\left(A B+t^{2}+A \sqrt{B^{2}+t^{2}}\right)^{\frac{1}{2 n}}}\right)
$$

since $B+|t| \geq \sqrt{B^{2}+t^{2}}$,

$$
\geq \frac{\theta\left|x_{j}\right|(2 n-1)\left(B^{2}+t^{2}\right)^{\frac{1}{2 n}}}{4 n N}\left(\frac{B^{2}+t^{2}}{A B+t^{2}+A \sqrt{B^{2}+t^{2}}}\right)^{\frac{1}{2 n}}
$$

Since $B^{2}+t^{2} \geq \frac{1}{4}\left(A B+t^{2}+A \sqrt{B^{2}+t^{2}}\right)$, then,

$$
\left|\partial_{x_{j}} N\right|+\theta\left|x_{j}\right|\left|\partial_{t} N\right| \geq(2 n-1) \frac{\theta\left|x_{j}\right|}{2^{\frac{1}{n}+2} n N} .
$$

For $j=1, n+1$, using the calculations (5.13) and (5.20),

$$
\begin{aligned}
& \left|\partial_{x_{j}} N\right|+\frac{\theta\left|x_{j}\right|}{2}\left|\partial_{t} N\right| \\
& =\frac{\theta\left|x_{j}\right|\left(B^{2}+t^{2}\right)^{\frac{1}{2 n}}}{4 n N} \\
& \left(\frac{\frac{1}{2} B^{2} A+\left(B-\frac{A}{2}\right) t^{2}+\frac{1}{2} \sqrt{B^{2}+t^{2}} A B+(n-1)\left(B^{2}+t^{2}\right)\left(B+\sqrt{B^{2}+t^{2}}\right)+n B \sqrt{B^{2}+t^{2}}\left(B+\sqrt{B^{2}+t^{2}}\right)}{\left(B+\sqrt{B^{2}+t^{2}}\right)\left(B^{2}+t^{2}\right)\left(A B+t^{2}+A \sqrt{B^{2}+t^{2}}\right)^{\frac{1}{2 n}}}\right) \\
& +\frac{\theta\left|x_{j}\right|\left(B^{2}+t^{2}\right)^{\frac{1}{2 n}}}{4 n N}\left(\frac{B(A-B)|t|+\frac{1}{2} A|t| \sqrt{B^{2}+t^{2}}+n|t| \frac{2 B^{3}+2 t^{2} B+2 B^{2} \sqrt{B^{2}+t^{2}}+t^{2} \sqrt{B^{2}+t^{2}}}{\left(B+\sqrt{B^{2}+t^{2}}\right)}}{\left(B+\sqrt{B^{2}+t^{2}}\right)\left(B^{2}+t^{2}\right)\left(A B+t^{2}+A \sqrt{B^{2}+t^{2}}\right)^{\frac{1}{2 n}}}\right) \\
& =\frac{\theta\left|x_{j}\right|\left(B^{2}+t^{2}\right)^{\frac{1}{2 n}}}{4 n N} \\
& \left(\frac{\frac{1}{2} B^{2} A+\left(B-\frac{A}{2}\right) t^{2}+\frac{1}{2} \sqrt{B^{2}+t^{2}} A B+(n-1)\left(B^{2}+t^{2}\right)\left(B+\sqrt{B^{2}+t^{2}}\right)+n B \sqrt{B^{2}+t^{2}}\left(B+\sqrt{B^{2}+t^{2}}\right)}{\left(B+\sqrt{B^{2}+t^{2}}\right)\left(B^{2}+t^{2}\right)\left(A B+t^{2}+A \sqrt{B^{2}+t^{2}}\right)^{\frac{1}{2 n}}}\right) \\
& +\frac{\theta\left|x_{j}\right|\left(B^{2}+t^{2}\right)^{\frac{1}{2 n}}}{4 n N}\left(\frac{B(A-B)|t|+\frac{1}{2} A|t| \sqrt{B^{2}+t^{2}}+n|t| \frac{2 B^{3}+2 t^{2} B+2 B^{2} \sqrt{B^{2}+t^{2}}+t^{2} \sqrt{B^{2}+t^{2}}}{\left(B+\sqrt{B^{2}+t^{2}}\right)}}{\left(B+\sqrt{B^{2}+t^{2}}\right)\left(B^{2}+t^{2}\right)\left(A B+t^{2}+A \sqrt{B^{2}+t^{2}}\right)^{\frac{1}{2 n}}}\right) \\
& =\frac{\theta\left|x_{j}\right|\left(B^{2}+t^{2}\right)^{\frac{1}{2 n}}}{4 n N} \\
& \left(\frac{\frac{1}{2} A B \sqrt{B^{2}+t^{2}}+\frac{1}{2} A|t| \sqrt{B^{2}+t^{2}}+\frac{A B^{2}}{2}+A B|t|-\frac{A t^{2}}{2}-B^{2} \sqrt{B^{2}+t^{2}}-t^{2} \sqrt{B^{2}+t^{2}}-B^{2}|t|-B^{3}}{\left(B+\sqrt{B^{2}+t^{2}}\right)\left(B^{2}+t^{2}\right)\left(A B+t^{2}+A \sqrt{B^{2}+t^{2}}\right)^{\frac{1}{2 n}}}\right) \\
& +\frac{\theta\left|x_{j}\right|\left(B^{2}+t^{2}\right)^{\frac{1}{2 n}}}{4 n N}\left(\frac{n\left(2 B^{2} \sqrt{B^{2}+t^{2}}+B|t| \sqrt{B^{2}+t^{2}}+t^{2} \sqrt{B^{2}+t^{2}}+B^{2}|t|+2 B^{3}+2 B t^{2}+|t|^{3}\right)}{\left(B+\sqrt{B^{2}+t^{2}}\right)\left(B^{2}+t^{2}\right)\left(A B+t^{2}+A \sqrt{B^{2}+t^{2}}\right)^{\frac{1}{2 n}}}\right)
\end{aligned}
$$




$$
\begin{aligned}
& \text { since } \frac{1}{2} A|t| \sqrt{B^{2}+t^{2}} \geq \frac{A t^{2}}{2}, A B|t| \geq B^{2}|t|, \text { and } \frac{1}{2} A B \sqrt{B^{2}+t^{2}}+\frac{A B^{2}}{2} \geq B^{3}, \\
& \geq \frac{\theta\left|x_{j}\right|\left(B^{2}+t^{2}\right)^{\frac{1}{2 n}}}{4 n N}\left(\frac{-B^{2} \sqrt{B^{2}+t^{2}}-t^{2} \sqrt{B^{2}+t^{2}}}{\left(B+\sqrt{B^{2}+t^{2}}\right)\left(B^{2}+t^{2}\right)\left(A B+t^{2}+A \sqrt{B^{2}+t^{2}}\right)^{\frac{1}{2 n}}}\right) \\
& +\frac{\theta\left|x_{j}\right|\left(B^{2}+t^{2}\right)^{\frac{1}{2 n}}}{4 n N}\left(\frac{n\left(2 B^{2} \sqrt{B^{2}+t^{2}}+B|t| \sqrt{B^{2}+t^{2}}+t^{2} \sqrt{B^{2}+t^{2}}+B^{2}|t|+2 B^{3}+2 B t^{2}+|t|^{3}\right)}{\left(B+\sqrt{B^{2}+t^{2}}\right)\left(B^{2}+t^{2}\right)\left(A B+t^{2}+A \sqrt{B^{2}+t^{2}}\right)^{\frac{1}{2 n}}}\right) \\
& =\frac{\theta\left|x_{j}\right|\left(B^{2}+t^{2}\right)^{\frac{1}{2 n}}}{4 n N}\left(\frac{(2 n-1) B^{2} \sqrt{B^{2}+t^{2}}+(n-1) t^{2} \sqrt{B^{2}+t^{2}}+n\left(B|t| \sqrt{B^{2}+t^{2}}+\left(B^{2}+t^{2}\right)(B+|t|+B)\right)}{\left(B+\sqrt{B^{2}+t^{2}}\right)\left(B^{2}+t^{2}\right)\left(A B+t^{2}+A \sqrt{B^{2}+t^{2}}\right)^{\frac{1}{2 n}}}\right) \\
& \geq \frac{\theta\left|x_{j}\right|\left(B^{2}+t^{2}\right)^{\frac{1}{2 n}}}{4 N}\left(\frac{\left(B^{2}+t^{2}\right)(B+|t|+B)}{\left(B+\sqrt{B^{2}+t^{2}}\right)\left(B^{2}+t^{2}\right)\left(A B+t^{2}+A \sqrt{B^{2}+t^{2}}\right)^{\frac{1}{2 n}}}\right)
\end{aligned}
$$

since $|t|+B \geq \sqrt{B^{2}+t^{2}}$,

$$
\geq \frac{\theta\left|x_{j}\right|}{4 N}\left(\frac{B^{2}+t^{2}}{A B+t^{2}+A \sqrt{B^{2}+t^{2}}}\right)^{\frac{1}{2 n}}
$$

Since $B^{2}+t^{2} \geq \frac{1}{4}\left(A B+t^{2}+A \sqrt{B^{2}+t^{2}}\right)$, then for $j=1, n+1$,

$$
\left|\partial_{x_{j}} N\right|+\frac{\theta\left|x_{j}\right|}{2}\left|\partial_{t} N\right| \geq \frac{\theta\left|x_{j}\right|}{2^{2+\frac{1}{n}} N} .
$$

Replacing (5.21) and (5.22) in (5.19) we get a lower bound for $|\nabla N|^{2}$ :

$$
\begin{aligned}
|\nabla N|^{2} \geq & \frac{1}{2}\left(\left|\partial_{x_{1}} N\right|+\frac{\theta\left|x_{1}\right|}{2}\left|\partial_{t} N\right|\right)^{2}+\frac{1}{2}\left(\left|\partial_{x_{n+1}} N\right|+\frac{\theta\left|x_{n+1}\right|}{2}\left|\partial_{t} N\right|\right)^{2} \\
& +\frac{1}{2} \sum_{j=2, j \neq n+1}^{2 n}\left(\left|\partial_{x_{j}} N\right|+\theta\left|x_{j}\right|\left|\partial_{t} N\right|\right)^{2} \\
\geq & \frac{\theta^{2}\left|x_{1}\right|^{2}}{2^{5+\frac{2}{n}} N^{2}}+\frac{\theta^{2}\left|x_{n+1}\right|^{2}}{2^{5+\frac{2}{n}} N^{2}}+\frac{\theta^{2}(2 n-1)^{2}}{2^{5+\frac{2}{n}} n^{2} N^{2}} \sum_{j=2, j \neq n+1}^{2 n}\left|x_{j}\right|^{2} .
\end{aligned}
$$

Hence, using the defined Euclidean norm $|x|=\left(\sum_{j=1}^{2 n} x_{j}^{2}\right)^{\frac{1}{2}}$,

$$
|\nabla N|^{2} \geq \frac{\theta^{2}|x|^{2}}{2^{5+\frac{2}{n}} N^{2}} .
$$


The last thing to do in this subsection is to obtain an upper bound for $|\nabla N|^{2}$. By (5.18),

$$
\begin{aligned}
|\nabla N|^{2} & =\sum_{j=1}^{2 n}\left(\partial_{x_{j}} N\right)^{2}+\frac{\theta^{2} x_{1}^{2}}{4}\left(\partial_{t} N\right)^{2}+\frac{\theta^{2} x_{n+1}^{2}}{4}\left(\partial_{t} N\right)^{2}+\sum_{j=2, j \neq n+1}^{2 n} \theta^{2} x_{j}^{2}\left(\partial_{t} N\right)^{2} \\
& \leq \sum_{j=1}^{2 n}\left(\partial_{x_{j}} N\right)^{2}+\theta^{2}|x|^{2}\left(\partial_{t} N\right)^{2}
\end{aligned}
$$

For $j=2, \ldots, n, n+2, \ldots, 2 n$, from (5.16) and the triangle inequality,

$$
\begin{aligned}
\left|\partial_{x_{j}} N\right|= & \frac{\theta\left|x_{j}\right|\left(B^{2}+t^{2}\right)^{\frac{1}{2 n}}}{4 n N} \\
& \cdot\left(\frac{A B \sqrt{B^{2}+t^{2}}+A B^{2}+(2 B-A) t^{2}+(2 n-1) B \sqrt{B^{2}+t^{2}}\left(B+\sqrt{B^{2}+t^{2}}\right)+t^{2} \sqrt{B^{2}+t^{2}}}{\left(B+\sqrt{B^{2}+t^{2}}\right)\left(B^{2}+t^{2}\right)\left(A B+t^{2}+A \sqrt{B^{2}+t^{2}}\right)^{\frac{1}{2 n}}}\right)
\end{aligned}
$$

since $\left(B^{2}+t^{2}\right) \leq\left(A B+t^{2}+A \sqrt{B^{2}+t^{2}}\right)$,

$$
\leq \frac{\theta\left|x_{j}\right|}{4 n N}\left(\frac{(2 n-1) B^{3}+(2 n-1) B^{2} \sqrt{B^{2}+t^{2}}+(2 n+1) B t^{2}+A B \sqrt{B^{2}+t^{2}}+A B^{2}-A t^{2}+t^{2} \sqrt{B^{2}+t^{2}}}{B^{3}+B^{2} \sqrt{B^{2}+t^{2}}+B t^{2}+t^{2} \sqrt{B^{2}+t^{2}}}\right)
$$

since $B \leq A \leq 2 B$,

$$
\leq \frac{\theta\left|x_{j}\right|}{4 n N}\left(\frac{(2 n+1) B^{3}+(2 n+1) B^{2} \sqrt{B^{2}+t^{2}}+(2 n) B t^{2}+t^{2} \sqrt{B^{2}+t^{2}}}{B^{3}+B^{2} \sqrt{B^{2}+t^{2}}+B t^{2}+t^{2} \sqrt{B^{2}+t^{2}}}\right) .
$$

So,

$$
\left|\partial_{x_{j}} N\right| \leq \frac{(2 n+1) \theta\left|x_{j}\right|}{4 n N}
$$

From (5.20),

$$
\left|\partial_{t} N\right|=\frac{|t|\left(B^{2}+t^{2}\right)^{\frac{1}{2 n}}}{4 n N}\left(\frac{2 B(A-B)+A \sqrt{B^{2}+t^{2}}+2 n \frac{2 B^{3}+2 t^{2} B+2 B^{2} \sqrt{B^{2}+t^{2}}+t^{2} \sqrt{B^{2}+t^{2}}}{\left(B+\sqrt{B^{2}+t^{2}}\right)}}{\left(B+\sqrt{B^{2}+t^{2}}\right)\left(B^{2}+t^{2}\right)\left(A B+t^{2}+A \sqrt{B^{2}+t^{2}}\right)^{\frac{1}{2 n}}}\right)
$$

since $\left(B^{2}+t^{2}\right) \leq\left(A B+t^{2}+A \sqrt{B^{2}+t^{2}}\right)$,

$$
\leq \frac{|t|}{4 n N}\left(\frac{2 B(A-B)+A \sqrt{B^{2}+t^{2}}+2 n \frac{2 B^{3}+2 t^{2} B+2 B^{2} \sqrt{B^{2}+t^{2}}+t^{2} \sqrt{B^{2}+t^{2}}}{\left(B+\sqrt{B^{2}+t^{2}}\right)}}{\left(B+\sqrt{B^{2}+t^{2}}\right)\left(B^{2}+t^{2}\right)}\right)
$$


since $|t| \leq \sqrt{B^{2}+t^{2}}$,

$$
\begin{aligned}
& \leq \frac{1}{4 n N}\left(\frac{\left(B+\sqrt{B^{2}+t^{2}}\right)\left(2 B(A-B)+A \sqrt{B^{2}+t^{2}}\right)+2 n\left(2 B^{3}+2 t^{2} B+2 B^{2} \sqrt{B^{2}+t^{2}}+t^{2} \sqrt{B^{2}+t^{2}}\right)}{\left(B+\sqrt{B^{2}+t^{2}}\right)^{2} \sqrt{B^{2}+t^{2}}}\right) \\
& =\frac{1}{4 n N}\left(\frac{3 B^{2} A-2 B^{3}+3 A B \sqrt{B^{2}+t^{2}}-2 B^{2} \sqrt{B^{2}+t^{2}}+A t^{2}+2 n\left(2 B^{3}+2 t^{2} B+2 B^{2} \sqrt{B^{2}+t^{2}}+t^{2} \sqrt{B^{2}+t^{2}}\right)}{2 B^{3}+2 t^{2} B+2 B^{2} \sqrt{B^{2}+t^{2}}+t^{2} \sqrt{B^{2}+t^{2}}}\right)
\end{aligned}
$$

since $A \leq \frac{B}{2}$

$$
\leq \frac{1}{4 n N}\left(\frac{-\frac{1}{2} B^{3}-\frac{1}{2} B^{2} \sqrt{B^{2}+t^{2}}+A t^{2}+2 n\left(2 B^{3}+2 t^{2} B+2 B^{2} \sqrt{B^{2}+t^{2}}+t^{2} \sqrt{B^{2}+t^{2}}\right)}{2 B^{3}+2 t^{2} B+2 B^{2} \sqrt{B^{2}+t^{2}}+t^{2} \sqrt{B^{2}+t^{2}}}\right) .
$$

Hence,

$$
\left|\partial_{t} N\right| \leq \frac{(2 n+1)}{4 n N}
$$

Inserting (5.15), (5.24), and (5.25) in (5.23), we get:

$$
\begin{aligned}
|\nabla N|^{2} & \leq \sum_{j=1}^{2 n}\left(\partial_{x_{j}} N\right)^{2}+\theta^{2}|x|^{2}\left(\partial_{t} N\right)^{2} \\
& \leq \frac{\theta^{2}\left|x_{1}\right|^{2}}{4 N^{2}}+\frac{\theta^{2}\left|x_{n+1}\right|^{2}}{4 N^{2}}+\sum_{j=2, j \neq n+1}^{2 n} \frac{(2 n+1)^{2} \theta^{2}\left|x_{j}\right|^{2}}{2^{4} n^{2} N^{2}}+\frac{(2 n+1)^{2} \theta^{2}}{2^{4} n^{2} N^{2}}|x|^{2} .
\end{aligned}
$$

So,

$$
|\nabla N|^{2} \leq \frac{(2 n+1)^{2} \theta^{2}|x|^{2}}{2^{3} n^{2} N^{2}}
$$

\section{References}

1. Bakry, D., Baudoin, F., Bonnefont, M., Chafai, D.: On gradient bounds for the heat kernel on the Heisenberg group. J. Funct. Anal. 255, 1905-1938 (2008)

2. Balogh, Z., Tyson, J.: Polar coordinates in Carnot groups. Math. Z. 241(4), 697-730 (2002)

3. Beals, R., Gaveau, B., Greiner, P.: The Green function of model step two hypoelliptic operators and the analysis of certain tangential Cauchy Riemann complexes. Adv. Math. 121, 288-345 (1996)

4. Bieske, T.: On the Lie algebra of polarizable Carnot groups. Anal. Math. Phys. 10, 80 (2020)

5. Bobkov, S., Zegarliński, B.: Entropy bounds and isoperimetry. Mem. Am. Math. Soc. 176(829), 1-67 (2005)

6. Bodineau, Th., Helffer, B.: On Log-Sobolev inequalities for unbounded spin systems. J. Funct. Anal. 166, 168-178 (1999)

7. Bonfiglioli, A., Lanconelli, E., Uguzzoni, F.: Stratified Lie Groups and Potential Theory for their Sub-Laplacians. Springer Monographs in Mathematics, Springer, Berlin (2007)

8. Bou Dagher, E., Zegarliński, B.: Coercive Inequalities and U-Bounds. arXiv:2105.01759 [math.FA] 
9. Brakalova, M., Markina, I., Vasil'ev, A.: Modules of systems of measures on polarizable Carnot groups. Ark. Mat. 54, 371-401 (2016)

10. Chatzakou, M., Federico, S., Zegarlinski, B.: q-Poincaré inequalities on Carnot Groups with a filiform Lie algebra. arXiv:2007.04689v2 [math.FA]

11. Cohn, W.S., Lu, G., Wang, P.: Sub-elliptic global high order Poincaré inequalities in stratified Lie groups and applications. (English summary). J. Funct. Anal. 249(2), 393-424 (2007)

12. D'Ambrosio, L.: Hardy type inequalities related to degenerate elliptic differential operators. Ann. Sc. Norm. Super. Pisa. Cl. Sci. (5) 4(3), 451-486 (2005)

13. Danielli, D., Garofalo, N., Phuc, N.C.: Hardy-Sobolev type inequalities with sharp constants in CarnotCarathéodory spaces. Potential Anal. 34, 223-242 (2011)

14. Folland, G.B.: Subelliptic estimates and function spaces on nilpotent Lie groups. Ark. Mat. 13(2), 161-207 (1975)

15. Goldstein, J.A., Kombe, I.: The Hardy inequality and nonlinear parabolic equations on Carnot groups. Nonlinear Anal. 69(12), 4643-4653 (2008)

16. Goldstein, J.A., Kombe, I., Yener, A.: A unified approach to weighted Hardy type inequalities on Carnot groups. Discrete Contin. Dyn. Syst. 37(4), 2009-2021 (2017)

17. Guionnet, A., Zegarliński, B.: Lectures on Logarithmic Sobolev Inequalities. Séminaire de Probabilités, XXXVI, Lecture Notes in Math., 1801, pp. 1-134. Springer, Berlin (2003)

18. Hebisch, W., Zegarliński, B.: Coercive inequalities on metric measure spaces. J. Funct. Anal. 258, 814-851 (2010)

19. Inglis, J.: Coercive Inequalities For Generators of Hörmander type. Doctor of Philosophy of the University of London and the Diploma of Imperial College, Department of Mathematics Imperial College (2010)

20. Inglis, J., Kontis, V., Zegarliński, B.: From U-Bounds to isoperimetry with applications. J. Funct. Anal. 260, 76-116 (2011)

21. Jerison, J.: The Poincaré inequality for vector fields satisfying Hörmander's condition. Duke Math. J. 53(2), 503-523 (1986)

22. Kombe, I.: Sharp weighted Rellich and uncertainty principle inequalities on Carnot groups. Commun. Appl. Anal. 14(2), 251-271 (2010)

23. Li, H.Q.: Estimation optimale du gradient du semi-groupe de la chaleur sur le groupe de Heisenberg. J. Funct. Anal. 236, 369-394 (2006)

24. Li, X., Lu, C.Z., Tang, H.L.: Poincaré inequalities for vector fields satisfying Hörmander's condition in variable exponent Sobolev spaces. Acta Math. Sin. (Engl. Ser.) 31(7), 1067-1085 (2015)

25. Lu, G.: Local and global interpolation inequalities on the Folland-Stein Sobolev spaces and polynomials on stratified groups. (English summary). Math. Res. Lett. 4(6), 777-790 (1997)

26. Lu, G.: Polynomials, higher order Sobolev extension theorems and interpolation inequalities on weighted Folland-Stein spaces on stratified groups. (English summary). Acta Math. Sin. (Engl. Ser.) 16(3), 405-444 (2000)

27. Lu, G., Wheeden, R.L.: High order representation formulas and embedding theorems on stratified groups and generalizations. Stud. Math. 142(2), 101-133 (2000). ((Reviewer: G. B. Folland))

28. Lu, G., Wheeden, R.L.: Simultaneous representation and approximation formula and high-order Sobolev embedding theorems on stratified groups. (English summary). Constr. Approx. 20(4), 647-668 (2004)

29. Ługiewicz, P., Zegarliński, B.: Coercive inequalities for Hörmander type generators in infinite dimensions. J. Funct. Anal. 247, 438-476 (2007)

30. Mechler, T.: Hypoelliptic heat kernel inequalities on Lie groups. Stoch. Process. Appl. 118(3), 368-388 (2008). ((Reviewer: T. Coulhon))

31. Roberto, C., Zegarliński, B.: Orlicz-Sobolev inequalities for sub-Gaussian measures and ergodicity of Markov semi-groups. J. Funct. Anal. 243, 28-66 (2006)

32. Ruzhansky, M., Yessirkegenov, N.: Factorization and Hardy-Rellich inequalities on stratified groups (2017). arXiv:1706.05108

33. Saloff-Coste, L.: Théorèmes de Sobolev et inégalités de Trudinger sur certain groupes de Lie. C. R. Acad. Sci. Paris 306, 305-308 (1988)

34. Varopoulos, N.T., Saloff-Coste, L., Coulhon, T.: Analysis and Geometry on Groups. Cambridge Tracts in Mathematics, 100, Cambridge University Press, Cambridge (1992)

35. Wang, J., Niu, P.: Sharp weighted Hardy type inequalities and Hardy-Sobolev type inequalities on polarizable Carnot groups. C. R. Math. Acad. Sci. Paris Ser. I 346, 1231-1234 (2008) 
36. Yang, Q.: Best constants in the Hardy-Rellich type inequalities on the Heisenberg group. J. Math. Anal. Appl. 342, 423-431 (2008)

37. Yosida, N.: The log-Sobolev inequality for weakly coupled lattice fields. Probab. Theor. Relat. Field 115, 1-40 (1999)

Publisher's Note Springer Nature remains neutral with regard to jurisdictional claims in published maps and institutional affiliations. 